\title{
Dynamic Analysis under Uniformly Distributed Moving Masses of Rectangular Plate with General Boundary Conditions
}

\author{
Emem Ayankop Andi ${ }^{1}$ and Sunday Tunbosun Oni $^{2}$ \\ ${ }^{1}$ Department of Mathematics, Nigerian Defence Academy, Kaduna State, Kaduna 800001, Nigeria \\ ${ }^{2}$ Department of Mathematical Sciences, Federal University of Technology, Ondo State, Akure 340001, Nigeria \\ Correspondence should be addressed to Emem Ayankop Andi; ememandi@yahoo.com
}

Received 3 December 2013; Revised 25 April 2014; Accepted 12 May 2014; Published 19 June 2014

Academic Editor: Nam-Il Kim

Copyright (c) 2014 E. A. Andi and S. T. Oni. This is an open access article distributed under the Creative Commons Attribution License, which permits unrestricted use, distribution, and reproduction in any medium, provided the original work is properly cited.

\begin{abstract}
The problem of the flexural vibrations of a rectangular plate having arbitrary supports at both ends is investigated. The solution technique which is suitable for all variants of classical boundary conditions involves using the generalized two-dimensional integral transform to reduce the fourth order partial differential equation governing the vibration of the plate to a second order ordinary differential equation which is then treated with the modified asymptotic method of Struble. The closed form solutions are obtained and numerical analyses in plotted curves are presented. It is also deduced that for the same natural frequency, the critical speed for the system traversed by uniformly distributed moving forces at constant speed is greater than that of the uniformly distributed moving mass problem for both clamped-clamped and simple-clamped end conditions. Hence resonance is reached earlier in the uniformly distributed moving mass system. Furthermore, for both structural parameters considered, the response amplitude of the moving distributed mass system is higher than that of the moving distributed force system. Thus, it is established that the moving distributed force solution is not an upper bound for an accurate solution of the moving distributed mass problem.
\end{abstract}

\section{Introduction}

The problem of assessing the dynamic behaviour of structures carrying moving loads has been almost exclusively reserved in the literature to that of one-dimensional structural members such as the response of railroad rails to moving trains, the response of bridges and elevated roadways to moving vehicles, the response of belt drives to conveyor belts, and the response of computer tape drives to floppy disks [1-8].

Where two-dimensional structures such as plates have been considered, the load acting on the structure has been simplified as lumped mass, point load, or concentrated load. Among researchers in this subject are Gbadeyan and Oni [9] who developed an analytical technique which is based on the generalized two-dimensional integral transform, the expression of the Dirac delta function as a Fourier Cosine series, and the use of the modified Struble's asymptotic method to solve the problem of rectangular plates under moving loads. This technique was later used by Oni [10] to solve the problem of the dynamic response of an elastic rectangular plate under the actions of several moving concentrated masses. Huang and Thambiratnam [11] studied isotropic homogenous elastic rectangular plate resting on an elastic Winkler foundation under a single concentrated load using finite strip method. Shadnam et al. [12] investigated the dynamics of plates under the influence of relatively large masses, moving along an arbitrary trajectory on the plate surface. In particular, a rectangular plate simply supported on all its edges was presented by means of operational calculus. Alisjahbana [13] presented an approximate method for the determination of the natural frequencies and mode shapes of rectangular clamped orthotropic plates subjected to dynamic transverse moving loads. The dynamic solution of the plate was based on orthogonality conditions of eigenfunctions. $\mathrm{Wu}$ et al. [14] analyzed by finite element method the dynamic responses of a flat plate subjected to various moving loads. The Newmark direct integration method was used to find the dynamic responses of the flat plate. Wang and Lin [15] presented the method of modal analysis to study the random vibration of multispan Mindlin plates due to load moving at a constant velocity. Although the above completed works on concentrated loads are impressive, they do not represent the 


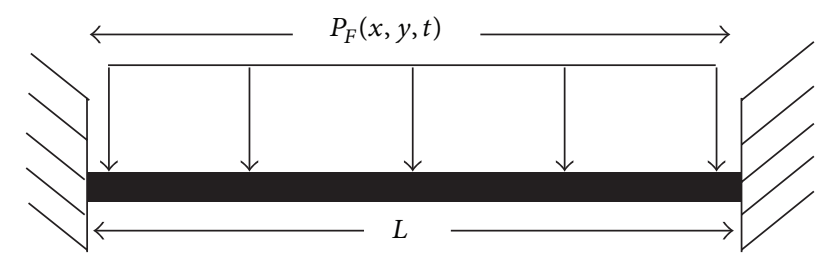

FIGURE 1: A uniformly distributed load $P_{F}(x, y, t)$ on a plate with both ends clamped.

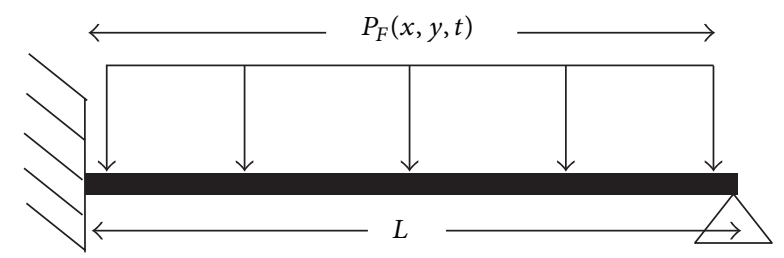

FIGURE 2: A uniformly distributed load $P_{F}(x, y, t)$ on a plate with one end clamped and the other end simply supported.

physical reality of the problem formulation as concentrated masses do not exist physically, since in practice moving loads are in the form of moving distributed masses which are actually distributed over a small segment or over the entire length of the structural member they traverse.

Works on 2-dimensional structural members traversed by distributed loads have only attracted the attention of few researchers (Figures 1 and 2 ).

These include the works of Gbadeyan and Dada [16] who investigated the dynamic response of plates on Pasternak foundation to distributed moving loads. Dada [17] who studied the vibration analysis of elastic plates under uniform partially distributed moving loads. Usman [18] discussed the dynamic response of a Bernoulli-beam on Winkler foundation under the action of partially distributed load. The dynamic analysis of Rayleigh beam carrying an added mass and traversed by uniform partially distributed moving loads was carried out by Adetunde [19]. More recently, Gbadeyan and Dada [20] used a finite difference algorithm to investigate the elastodynamic response of a rectangular Mindlin plate subjected to a distributed moving mass. The simply supported edge condition was used as an illustrative example. It was found that the maximum shearing forces, bending, and twisting moments occur almost at the same time. Beskou and Theodorakopoulos [21] presented a review of the dynamic response of road pavements modelled as a beam, plate, or top layer of a layered soil medium to moving loads on their surface. The loads were concentrated or distributed of finite extent, varied with time, and moved with constant or variable speed. The analysis was done by analytical/numerical and purely numerical methods, such as finite element and boundary element methods, under conditions of plane strain. Representative examples were presented in order to illustrate the problem. Amiri et al. [22] investigated the elastodynamic response of a rectangular Mindlin plate under concentrated moving loads as well as other arbitrarily selected distribution area of loads. Closed form solution for the moving force load case was derived using direct separation of variables and eigenfunction expansion method. A semianalytical solution was presented for moving mass load case. In these investigations, however, only numerical or semianalytical techniques have been employed to solve the governing equation due to the rigour of the loadstructure interactions and complex nature of the resulting equations. Nevertheless, analytical solution is desirable as it often sheds light on some vital information in the vibrating system. This paper therefore investigates the flexural vibrations of a rectangular plate with general boundary conditions under uniformly distributed moving masses. Both gravity and inertia effects of the uniformly distributed masses are taken into consideration, and the plate is taken to rest on Winkler foundation. The solution technique which is analytical and suitable for all variants of classical boundary conditions involves using the generalized two-dimensional integral transform, the expression of the Heaviside function as a Fourier series and the use of the modified Struble's asymptotic technique to solve the problem of the flexural vibrations of a rectangular plate having arbitrary supports at both ends. In addition, conditions under which resonance is reached, is obtained for both uniformly distributed moving force and uniformly distributed moving mass problems.

\section{Governing Equation}

Consider the dynamic transverse displacement $W(x, y, t)$ of a rectangular flat plate of span $L_{y}$ along the $y$-axis and span $L_{x}$ along the $x$-axis carrying a uniformly distributed mass moving with constant velocity $c$ along a straight line $y=y_{0}$ parallel to the $x$-axis.

If the plate model incorporates the rotatory inertia correction factor, $W(x, y, t)$ is governed by the fourth order partial differential equation given by [23]

$$
\begin{aligned}
& \left(D\left(\frac{\partial^{2}}{\partial x^{2}}+\frac{\partial^{2}}{\partial y^{2}}\right)^{2}-\mu R_{0} \frac{\partial^{2}}{\partial t^{2}}\right)\left(\frac{\partial^{2}}{\partial x^{2}}+\frac{\partial^{2}}{\partial y^{2}}\right)^{2} \\
& \quad \times W(x, y, t)+\mu \frac{\partial^{2} W(x, y, t)}{\partial t^{2}}+K W(x, y, t) \\
& =P_{F}(x, y, t)\left[1-\frac{\Delta^{*}}{g}[W(x, y, t)]\right] .
\end{aligned}
$$

$E$ is Young's modulus, $\nu$ is Poisson's ratio $(\nu<1), \mu$ is the mass per unit length (self-weight) of the plate, $x$ is the position coordinate in $x$-direction, $y$ is the position coordinate in $y$ direction, $t$ is the time, $h$ is the plate thickness, $K$ is the elastic foundation moduli, and $R_{0}$ is the measure of rotatory inertia correction effect. $D=E h^{3} / 12(1-v)$ is the bending rigidity of the plate and is constant throughout the plate. $P_{F}(x, y, t)$ is the continuous moving uniformly distributed force acting on the beam model. $g$ is acceleration due to gravity and $\Delta^{*}$ is the convective acceleration operator defined as

$$
\Delta^{*}=\left(\frac{\partial^{2}}{\partial t^{2}}+2 c \frac{\partial^{2}}{\partial t \partial x}+c^{2} \frac{\partial^{2}}{\partial x^{2}}\right) \text {. }
$$


The continuous moving uniformly distributed force acting on the plate is given by

$$
P_{F}(x, y, t)=\operatorname{MgH}(x-c t) H\left(y-y_{0}\right),
$$

where $M$ is the uniformly distributed mass, $H(\cdot)$ is the Heaviside function. The time $t$ is assumed to be limited to that interval of time within which the mass $M$ is on the plate; that is,

$$
0 \leq c t \leq L_{x}
$$

Thus, in view of (2) and (3), (1) can be written as

$$
\begin{aligned}
& D\left[\frac{\partial^{4} W(x, y, t)}{\partial x^{4}}+2 \frac{\partial^{4} W(x, y, t)}{\partial x^{2} \partial y^{2}}+\frac{\partial^{4} W(x, y, t)}{\partial y^{4}}\right] \\
& \quad+\mu \frac{\partial^{2} W(x, y, t)}{\partial t^{2}}+K W(x, y, t) \\
& \quad-\mu R_{0}\left[\frac{\partial^{4} W(x, y, t)}{\partial x^{2} \partial t^{2}}+\frac{\partial^{4} W(x, y, t)}{\partial y^{2} \partial t^{2}}\right] \\
& \quad+M H(x-c t) H\left(y-y_{0}\right) \\
& \quad \times\left[\frac{\partial^{2} W(x, y, t)}{\partial t^{2}}+2 c \frac{\partial^{2} W(x, y, t)}{\partial t \partial x}+c^{2} \frac{\partial^{2} W(x, y, t)}{\partial x^{2}}\right] \\
& =M g H(x-c t) H\left(y-y_{0}\right) .
\end{aligned}
$$

Equation (5) is the fourth order partial differential equation governing the flexural vibrations of a rectangular plate under the action of uniformly distributed loads. The boundary conditions are taken to be arbitrary, while the initial conditions without any loss of generality are given by

$$
W(x, y, 0)=0=\frac{\partial W(x, y, 0)}{\partial t} .
$$

\section{Method of Solution}

The analysis of the dynamic response to a uniformly distributed moving mass of isotropic rectangular plates resting on a Winkler foundation and subjected to arbitrary boundary conditions is carried out in this section. The generalized twodimensional integral transform is defined as

$$
\bar{W}(j, k, t)=\int_{0}^{L_{y}} \int_{0}^{L_{x}} W(x, y, t) W_{j}(x) W_{k}(y) d x d y
$$

with the inverse

$$
W(x, y, t)=\sum_{j=1}^{\infty} \sum_{k=1}^{\infty} \frac{\mu}{V_{j}} \frac{\mu}{V_{k}} \bar{W}(j, k, t) W_{j}(x) W_{k}(y),
$$

where

$$
V_{j}=\int_{0}^{L_{x}} \mu W_{j}^{2}(x) d x, \quad V_{k}=\int_{0}^{L_{y}} \mu W_{k}^{2}(y) d y .
$$

$W_{j}(x)$ and $W_{k}(y)$ are, respectively, the beam mode functions in the $x$ and $y$ directions defined, respectively, as

$$
\begin{aligned}
W_{j}(x)= & \operatorname{Sin} \frac{\lambda_{j} x}{L_{x}}+A_{j} \operatorname{Cos} \frac{\lambda_{j} x}{L_{x}} \\
& +B_{j} \operatorname{Sin} h \frac{\lambda_{j} x}{L_{x}}+C_{j} \operatorname{Cos} h \frac{\lambda_{j} x}{L_{x}}, \\
W_{k}(y)= & \operatorname{Sin} \frac{\lambda_{k} y}{L_{y}}+A_{k} \operatorname{Cos} \frac{\lambda_{k} y}{L_{y}} \\
& +B_{k} \operatorname{Sin} h \frac{\lambda_{k} y}{L_{y}}+C_{k} \operatorname{Cosh} \frac{\lambda_{k} y}{L_{y}},
\end{aligned}
$$

where, $A_{j}, B_{j}, C_{j}, A_{k}, B_{k}$, and $C_{k}$ are constants and $\lambda_{j}$, $\lambda_{k}$ are mode frequencies which are determined using the appropriate boundary conditions.

Applying the generalized two-dimensional integral transform (7), (5) takes the form

$$
\begin{aligned}
Z_{A} F_{1} & \left(0, L_{x}, L_{y}, t\right)+Z_{A} F_{2}\left(0, L_{x}, L_{y}, t\right)+\bar{W}_{t t}(j, k, t) \\
& -Z_{B} T_{B 1}(t)-Z_{B} T_{B 2}(t)+Z_{C} \bar{W}(j, k, t) \\
& +T_{B 3}(t)+T_{B 4}(t)+T_{B 5}(t) \\
= & \frac{M g}{\mu} \int_{0}^{L_{y}} \int_{0}^{L_{x}} H(x-c t) H\left(y-y_{0}\right) W_{j}(x) W_{k}(y) d x d y,
\end{aligned}
$$

where

$$
Z_{A}=\frac{D}{\mu}, \quad Z_{B}=R_{0}, \quad Z_{C}=\frac{K}{\mu},
$$

$$
\begin{aligned}
& F_{1}\left(0, L_{x}, L_{y}, t\right) \\
&=\int_{0}^{L_{y}}\left(\left[W_{j}(x) \frac{\partial^{3} W(x, y, t)}{\partial x^{3}}-W_{j}^{\prime}(x) \frac{\partial^{2} W(x, y, t)}{\partial x^{2}}\right.\right. \\
&\left.+W_{j}^{\prime \prime}(x) \frac{\partial W(x, y, t)}{\partial x}-W_{j}^{\prime \prime \prime}(x) W(x, y, t)\right]_{0}^{L_{x}} \\
& \quad \times W_{k}(y) d y \\
&+2\left[W_{j}(x) \frac{\partial W(x, y, t)}{\partial x}-W_{j}^{\prime}(x) W(x, y, t)\right]^{\prime \prime} \\
&\left.\times W_{k}^{\prime \prime}(y) d y\right)
\end{aligned}
$$

$$
+\int_{0}^{L_{x}}\left(2\left[W_{k}(y) \frac{\partial W(x, y, t)}{\partial y}-W_{k}^{\prime}(y) W(x, y, t)\right]_{0}^{L_{y}}\right.
$$




$$
\begin{aligned}
& \times W_{j}^{\prime \prime}(x) d x \\
& +\left[W_{k}(y) \frac{\partial^{3} W(x, y, t)}{\partial y^{3}}\right. \\
& -W_{k}^{\prime}(y) \frac{\partial^{2} W(x, y, t)}{\partial y^{2}}+W_{k}^{\prime \prime}(y) \frac{\partial W(x, y, t)}{\partial y} \\
& \left.\left.-W_{k}^{\prime \prime \prime}(y) W(x, y, t)\right]_{0}^{L_{y}} W_{j}(x) d x\right), \\
& F_{2}\left(0, L_{x}, L_{y}, t\right) \\
& =\int_{0}^{L_{y}} \int_{0}^{L_{x}}\left(W(x, y, t) W_{j}^{\prime v}(x) W_{k}(y)\right. \\
& \left.+2 W(x, y, t) W_{j}^{\prime \prime}(x) W_{k}^{\prime \prime}(y)\right) d x d y \\
& +\int_{0}^{L_{y}} \int_{0}^{L_{x}} W(x, y, t) W_{j}(x) W_{k}^{\prime v}(y) d x d y, \\
& T_{B 1}(t)=\int_{0}^{L_{y}} \int_{0}^{L_{x}} \frac{\partial^{4} W(x, y, t)}{\partial t^{2} \partial x^{2}} W_{j}(x) W_{k}(y) d x d y, \\
& T_{B 2}(t)=\int_{0}^{L_{y}} \int_{0}^{L_{x}} \frac{\partial^{4} W(x, y, t)}{\partial t^{2} \partial y^{2}} W_{j}(x) W_{k}(y) d x d y, \\
& T_{B 3}(t)=\frac{M}{\mu} \int_{0}^{L_{y}} \int_{0}^{L_{x}} H(x-c t) H\left(y-y_{0}\right) \\
& \times \frac{\partial^{2} W(x, y, t)}{\partial t^{2}} W_{j}(x) W_{k}(y) d x d y, \\
& T_{B 4}(t)=2 \frac{M c}{\mu} \int_{0}^{L_{y}} \int_{0}^{L_{x}} H(x-c t) H\left(y-y_{0}\right) \\
& \times \frac{\partial^{2} W(x, y, t)}{\partial t \partial x} W_{j}(x) W_{k}(y) d x d y, \\
& T_{B 5}(t)=\frac{M c^{2}}{\mu} \int_{0}^{L_{y}} \int_{0}^{L_{x}} H(x-c t) H\left(y-y_{0}\right) \\
& \times \frac{\partial^{2} W(x, y, t)}{\partial x^{2}} W_{j}(x) W_{k}(y) d x d y .
\end{aligned}
$$

It is recalled that the equation of the free vibration of a rectangular plate is given by

$$
\begin{aligned}
& D\left[\frac{\partial^{4} W(x, y, t)}{\partial x^{4}}+2 \frac{\partial^{4} W(x, y, t)}{\partial x^{2} \partial y^{2}}+\frac{\partial^{4} W(x, y, t)}{\partial y^{4}}\right] \\
& \quad+\mu \frac{\partial^{2} W(x, y, t)}{\partial t^{2}}=0 .
\end{aligned}
$$

If the free vibration solution of the problem is set as

$$
W(x, y, t)=W_{j}(x) W_{k}(y) \operatorname{Sin} \Omega_{j, k} t,
$$

where $\Omega_{j, k}$ is the natural circular frequency of a rectangular plate, then substituting (21) into (20) yields

$$
\begin{aligned}
D & {\left[W_{j}^{\prime v}(x) W_{k}(y)+2 W_{j}^{\prime \prime}(x) W_{k}^{\prime \prime}(y)+W_{j}(x) W_{k}^{\prime v}(y)\right] } \\
& -\mu \Omega_{j, k}^{2} W_{j}(x) W_{k}(y)=0 .
\end{aligned}
$$

It is well known that for a simply supported rectangular plate, $\Omega_{j, k}^{2}$ is given by

$$
\Omega_{j, k}^{2}=D\left[\frac{j^{4} \pi^{4}}{L_{x}^{4}}+2 \frac{j^{2} k^{2} \pi^{4}}{L_{x}^{2} L_{y}^{2}}+\frac{k^{4} \pi^{4}}{L_{y}^{4}}\right] .
$$

Multiplying (22) by $W(x, y, t)$ and integrating with respect to $x$ and $y$ between the limits $0, L_{x}$ and $0, L_{y}$, respectively, we get

$$
\begin{aligned}
& \int_{0}^{L_{y}} \int_{0}^{L_{x}} W(x, y, t) W_{j}^{\prime v}(x) W_{k}(y) d x d y \\
& \quad+2 \int_{0}^{L_{y}} \int_{0}^{L_{x}} W(x, y, t) W_{j}^{\prime \prime}(x) W_{k}^{\prime \prime}(y) d x d y \\
& \quad+\int_{0}^{L_{y}} \int_{0}^{L_{x}} W(x, y, t) W_{j}(x) W_{k}^{\prime v}(y) d x d y \\
& =\frac{\mu}{D} \Omega_{j, k}^{2} \int_{0}^{L_{y}} \int_{0}^{L_{x}} W(x, y, t) W_{j}(x) W_{k}(y) d x d y .
\end{aligned}
$$

In view of (14) we have

$$
F_{2}\left(0, L_{x}, L_{y}\right)=\frac{\mu}{D} \Omega_{j, k}^{2} \bar{W}(j, k, t) .
$$

Since $\bar{W}(p, q, t)$ is just the coefficient of the generalized twodimensional finite integral transform

$$
W(x, y, t)=\sum_{p=1}^{\infty} \sum_{q=1}^{\infty} \frac{\mu}{V_{p}} \frac{\mu}{V_{q}} \bar{W}(p, q, t) W_{p}(x) W_{q}(y),
$$

it follows that

$$
W^{\prime \prime}(x, y, t)=\sum_{p=1}^{\infty} \sum_{q=1}^{\infty} \frac{\mu}{V_{p}} \frac{\mu}{V_{q}} \bar{W}(p, q, t) W_{p}^{\prime \prime}(x) W_{q}(y) .
$$

Therefore, the integrals (15) and (16) can be rewritten as

$$
T_{B 1}(t)=\sum_{p=1}^{\infty} \sum_{q=1}^{\infty} \frac{\mu^{2}}{V_{p} V_{q}} \bar{W}_{t t}(p, q, t) H_{2}(p, j) F(q, k),
$$

where

$$
\begin{gathered}
H_{2}(p, j)=\int_{0}^{L_{x}} W_{p}^{\prime \prime}(x) W_{j}(x) d x, \\
F(q, k)=\int_{0}^{L_{y}} W_{q}(y) W_{k}(y) d y, \\
T_{B 2}(t)=\sum_{p=1}^{\infty} \sum_{q=1}^{\infty} \frac{\mu^{2}}{V_{p} V_{q}} \bar{W}_{t t}(p, q, t) H(p, j) F_{2}(q, k),
\end{gathered}
$$


where

$$
\begin{gathered}
H(p, j)=\int_{0}^{L_{x}} W_{p}(x) W_{j}(x) d x, \\
F_{2}(q, k)=\int_{0}^{L_{y}} W_{q}^{\prime \prime}(y) W_{k}(y) d y .
\end{gathered}
$$

In order to evaluate the integrals (17), (18), and (19), use is made of the Fourier series representation of the Heaviside function; namely,

$$
H(x-c t)=\frac{1}{4}+\frac{1}{\pi} \sum_{n=0}^{\infty} \frac{\operatorname{Sin}((2 n+1) \pi(x-c t))}{2 n+1} .
$$

Similarly,

$$
H\left(y-y_{0}\right)=\frac{1}{4}+\frac{1}{\pi} \sum_{m=0}^{\infty} \frac{\operatorname{Sin}\left((2 m+1) \pi\left(y-y_{0}\right)\right)}{2 m+1} .
$$

Using (30) and (31), one obtains

$$
\begin{aligned}
& T_{B 3}(t) \\
& =\frac{M}{16 \mu} \sum_{p=1}^{\infty} \sum_{q=1}^{\infty} \frac{\mu^{2}}{V_{p} V_{q}} \bar{W}_{t t}(p, q, t) F(q, k) H(p, j) \\
& +\frac{M}{4 \mu \pi} \sum_{p=1}^{\infty} \sum_{q=1}^{\infty} \sum_{n=0}^{\infty} \frac{\mu^{2}}{V_{p} V_{q}} \bar{W}_{t t}(p, q, t) \\
& \times \frac{\operatorname{Cos}(2 n+1) \pi c t}{2 n+1} H^{s}(n, p, j) F(q, k) \\
& -\frac{M}{4 \mu \pi} \sum_{p=1}^{\infty} \sum_{q=1}^{\infty} \sum_{n=0}^{\infty} \frac{\mu^{2}}{V_{p} V_{q}} \bar{W}_{t t}(p, q, t) \\
& \times \frac{\operatorname{Sin}(2 n+1) \pi c t}{2 n+1} H^{c}(n, p, j) F(q, k) \\
& +\frac{M}{4 \mu \pi} \sum_{p=1}^{\infty} \sum_{q=1}^{\infty} \sum_{n=0}^{\infty} \frac{\mu^{2}}{V_{p} V_{q}} \bar{W}_{t t}(p, q, t) \\
& \times \frac{\operatorname{Cos}(2 m+1) \pi y_{0}}{2 m+1} F^{s}(m, q, k) H(p, j) \\
& -\frac{M}{4 \mu \pi} \sum_{p=1}^{\infty} \sum_{q=1}^{\infty} \sum_{n=0}^{\infty} \frac{\mu^{2}}{V_{p} V_{q}} \bar{W}_{t t}(p, q, t) \\
& \times \frac{\operatorname{Sin}(2 m+1) \pi y_{0}}{2 m+1} F^{c}(m, q, k) H(p, j)
\end{aligned}
$$

$$
\begin{aligned}
&+\frac{M}{\mu \pi^{2}} \sum_{p=1}^{\infty} \sum_{q=1}^{\infty} \sum_{n=0}^{\infty} \sum_{m=0}^{\infty} \frac{\mu^{2}}{V_{p} V_{q} \bar{W}_{t t}(p, q, t)} \times \frac{\operatorname{Cos}(2 n+1) \pi c t}{2 n+1} \\
& \cdot \frac{\operatorname{Cos}(2 m+1) \pi y_{0}}{2 m+1} \\
& \times H^{s}(n, p, j) F^{s}(m, q, k) \\
&-\frac{M}{\mu \pi^{2}} \sum_{p=1}^{\infty} \sum_{q=1}^{\infty} \sum_{n=0}^{\infty} \sum_{m=0}^{\infty} \frac{\mu^{2}}{V_{p} V_{q}} \bar{W}_{t t}(p, q, t) \\
& \times \frac{\operatorname{Cos}(2 n+1) \pi c t}{2 n+1} \\
& \cdot \frac{\operatorname{Sin}(2 m+1) \pi y_{0}}{2 m+1} \\
& \times H^{s}(n, p, j) F^{c}(m, q, k)
\end{aligned}
$$

$$
\begin{aligned}
-\frac{M}{\mu \pi^{2}} \sum_{p=1}^{\infty} \sum_{q=1}^{\infty} \sum_{n=0}^{\infty} \sum_{m=0}^{\infty} & \frac{\mu^{2}}{V_{p} V_{q}} \bar{W}_{t t}(p, q, t) \\
& \times \frac{\operatorname{Sin}(2 n+1) \pi c t}{2 n+1} \\
& \cdot \frac{\operatorname{Cos}(2 m+1) \pi y_{0}}{2 m+1} \\
& \times H^{c}(n, p, j) F^{s}(m, q, k)
\end{aligned}
$$$$
+\frac{M}{\mu \pi^{2}} \sum_{p=1}^{\infty} \sum_{q=1}^{\infty} \sum_{n=0}^{\infty} \sum_{m=0}^{\infty} \frac{\mu^{2}}{V_{p} V_{q}} \bar{W}_{t t}(p, q, t)
$$$$
\times \frac{\operatorname{Sin}(2 n+1) \pi c t}{2 n+1}
$$$$
\cdot \frac{\operatorname{Sin}(2 m+1) \pi y_{0}}{2 m+1}
$$$$
\times H^{c}(n, p, j) F^{c}(m, q, k) \text {, }
$$

where

$$
\begin{aligned}
& H^{s}(n, p, j)=\int_{0}^{L_{x}} \operatorname{Sin}(2 n+1) \pi x W_{p}(x) W_{j}(x) d x, \\
& H^{c}(n, p, j)=\int_{0}^{L_{x}} \operatorname{Cos}(2 n+1) \pi x W_{p}(x) W_{j}(x) d x, \\
& F^{s}(m, q, k)=\int_{0}^{L_{y}} \operatorname{Sin}(2 m+1) \pi y W_{q}(y) W_{k}(y) d y, \\
& F^{c}(m, q, k)=\int_{0}^{L_{y}} \operatorname{Cos}(2 m+1) \pi y W_{q}(y) W_{k}(y) d y .
\end{aligned}
$$


Similarly,

$$
\begin{aligned}
& T_{B 4}(t) \\
& =\frac{2 M c}{16 \mu} \sum_{p=1}^{\infty} \sum_{q=1}^{\infty} \frac{\mu^{2}}{V_{p} V_{q}} \bar{W}_{t}(p, q, t) H_{1}(p, j) F(q, k) \\
& +\frac{2 M c}{4 \mu \pi} \sum_{p=1}^{\infty} \sum_{q=1}^{\infty} \sum_{n=0}^{\infty} \frac{\mu^{2}}{V_{p} V_{q}} \bar{W}_{t}(p, q, t) \\
& \times \frac{\operatorname{Cos}(2 n+1) \pi c t}{2 n+1} H_{1}^{s}(n, p, j) F(q, k) \\
& -\frac{2 M c}{4 \mu \pi} \sum_{p=1}^{\infty} \sum_{q=1}^{\infty} \sum_{n=0}^{\infty} \frac{\mu^{2}}{V_{p} V_{q}} \bar{W}_{t}(p, q, t) \\
& \times \frac{\operatorname{Sin}(2 n+1) \pi c t}{2 n+1} H_{1}^{c}(n, p, j) F(q, k) \\
& +\frac{2 M c}{4 \mu \pi} \sum_{p=1}^{\infty} \sum_{q=1}^{\infty} \sum_{n=0}^{\infty} \frac{\mu^{2}}{V_{p} V_{q}} \bar{W}_{t}(p, q, t) \frac{\operatorname{Cos}(2 m+1) \pi y_{0}}{2 m+1} \\
& \times F^{s}(m, q, k) H_{1}(p, j) \\
& -\frac{2 M c}{4 \mu \pi} \sum_{p=1}^{\infty} \sum_{q=1}^{\infty} \sum_{n=0}^{\infty} \frac{\mu^{2}}{V_{p} V_{q}} \bar{W}_{t}(p, q, t) \frac{\operatorname{Sin}(2 m+1) \pi y_{0}}{2 m+1} \\
& \times F^{c}(m, q, k) H_{1}(p, j) \\
& +\frac{2 M c}{\mu \pi^{2}} \sum_{p=1}^{\infty} \sum_{q=1}^{\infty} \sum_{n=0}^{\infty} \sum_{m=0}^{\infty} \frac{\mu^{2}}{V_{p} V_{q}} \bar{W}_{t}(p, q, t) \frac{\operatorname{Cos}(2 n+1) \pi c t}{2 n+1} \\
& \cdot \frac{\operatorname{Cos}(2 m+1) \pi y_{0}}{2 m+1} \\
& \times H_{1}^{s}(n, p, j) F^{s}(m, q, k) \\
& -\frac{2 M c}{\mu \pi^{2}} \sum_{p=1}^{\infty} \sum_{q=1}^{\infty} \sum_{n=0}^{\infty} \sum_{m=0}^{\infty} \frac{\mu^{2}}{V_{p} V_{q}} \bar{W}_{t}(p, q, t) \frac{\operatorname{Cos}(2 n+1) \pi c t}{2 n+1} \\
& \frac{\operatorname{Sin}(2 m+1) \pi y_{0}}{2 m+1} \\
& \times H_{1}^{s}(n, p, j) F^{c}(m, q, k) \\
& \begin{aligned}
-\frac{2 M c}{\mu \pi^{2}} \sum_{p=1}^{\infty} \sum_{q=1}^{\infty} \sum_{n=0}^{\infty} \sum_{m=0}^{\infty} & \frac{\mu^{2}}{V_{p} V_{q}} \bar{W}_{t}(p, q, t) \frac{\operatorname{Sin}(2 n+1) \pi c t}{2 n+1} \\
& \cdot \frac{\operatorname{Cos}(2 m+1) \pi y_{0}}{2 m+1} \\
& \times H_{1}^{c}(n, p, j) F^{s}(m, q, k)
\end{aligned} \\
& +\frac{2 M c}{\mu \pi^{2}} \sum_{p=1}^{\infty} \sum_{q=1}^{\infty} \sum_{n=0}^{\infty} \sum_{m=0}^{\infty} \frac{\mu^{2}}{V_{p} V_{q}} \bar{W}_{t}(p, q, t) \frac{\operatorname{Sin}(2 n+1) \pi c t}{2 n+1} \\
& \begin{array}{l}
\cdot \frac{\operatorname{Sin}(2 m+1) \pi y_{0}}{2 m+1} \\
\times H_{1}^{c}(n, p, j) F^{c}(m, q, k),
\end{array}
\end{aligned}
$$

where

$$
\begin{aligned}
& H_{1}(p, j)=\int_{0}^{L_{x}} W_{p}^{\prime}(x) W_{j}(x) d x, \\
& H_{1}^{s}(n, p, j)=\int_{0}^{L_{x}} \operatorname{Sin}(2 n+1) \pi x W_{p}^{\prime}(x) W_{j}(x) d x, \\
& H_{1}^{c}(n, p, j)=\int_{0}^{L_{x}} \operatorname{Cos}(2 n+1) \pi x W_{p}^{\prime}(x) W_{j}(x) d x, \\
& T_{B 5}(t) \\
& =\frac{M c^{2}}{16 \mu} \sum_{p=1}^{\infty} \sum_{q=1}^{\infty} \frac{\mu^{2}}{V_{p} V_{q}} \bar{W}(p, q, t) H_{2}(p, j) F(q, k) \\
& +\frac{M c^{2}}{4 \mu \pi} \sum_{p=1}^{\infty} \sum_{q=1}^{\infty} \sum_{n=0}^{\infty} \frac{\mu^{2}}{V_{p} V_{q}} \bar{W}_{t}(p, q, t) \\
& \times \frac{\operatorname{Cos}(2 n+1) \pi c t}{2 n+1} H_{2}^{s}(n, p, j) F(q, k) \\
& -\frac{M c^{2}}{4 \mu \pi} \sum_{p=1}^{\infty} \sum_{q=1}^{\infty} \sum_{n=0}^{\infty} \frac{\mu^{2}}{V_{p} V_{q}} \bar{W}_{t}(p, q, t) \\
& \times \frac{\operatorname{Sin}(2 n+1) \pi c t}{2 n+1} H_{2}^{c}(n, p, j) F(q, k) \\
& +\frac{M c^{2}}{4 \mu \pi} \sum_{p=1}^{\infty} \sum_{q=1}^{\infty} \sum_{n=0}^{\infty} \frac{\mu^{2}}{V_{p} V_{q}} \bar{W}_{t}(p, q, t) \\
& \times \frac{\operatorname{Cos}(2 m+1) \pi y_{0}}{2 m+1} \\
& \times F^{s}(m, q, k) H_{2}(p, j) \\
& -\frac{M c^{2}}{4 \mu \pi} \sum_{p=1}^{\infty} \sum_{q=1}^{\infty} \sum_{n=0}^{\infty} \frac{\mu^{2}}{V_{p} V_{q}} \bar{W}_{t}(p, q, t) \\
& \times \frac{\operatorname{Sin}(2 m+1) \pi y_{0}}{2 m+1} \\
& \times F^{c}(m, q, k) H_{2}(p, j) \\
& +\frac{M c^{2}}{\mu \pi^{2}} \sum_{p=1}^{\infty} \sum_{q=1}^{\infty} \sum_{n=0}^{\infty} \sum_{m=0}^{\infty} \frac{\mu^{2}}{V_{p} V_{q}} \bar{W}_{t}(p, q, t) \frac{\operatorname{Cos}(2 n+1) \pi c t}{2 n+1} \\
& \frac{\operatorname{Cos}(2 m+1) \pi y_{0}}{2 m+1} \\
& \times H_{2}^{s}(n, p, j) F^{s}(m, q, k) \\
& -\frac{M c^{2}}{\mu \pi^{2}} \sum_{p=1}^{\infty} \sum_{q=1}^{\infty} \sum_{n=0}^{\infty} \sum_{m=0}^{\infty} \frac{\mu^{2}}{V_{p} V_{q}} \bar{W}_{t}(p, q, t) \frac{\operatorname{Cos}(2 n+1) \pi c t}{2 n+1} \\
& \frac{\operatorname{Sin}(2 m+1) \pi y_{0}}{2 m+1} \\
& \times H_{2}^{s}(n, p, j) F^{c}(m, q, k)
\end{aligned}
$$




$$
\begin{aligned}
&-\frac{M c^{2}}{\mu \pi^{2}} \sum_{p=1}^{\infty} \sum_{q=1}^{\infty} \sum_{n=0}^{\infty} \sum_{m=0}^{\infty} \frac{\mu^{2}}{V_{p} V_{q}} \bar{W}_{t}(p, q, t) \frac{\operatorname{Sin}(2 n+1) \pi c t}{2 n+1} \\
& \cdot \frac{\operatorname{Cos}(2 m+1) \pi y_{0}}{2 m+1} \\
& \times H_{2}^{c}(n, p, j) F^{s}(m, q, k) \\
&+\frac{M c^{2}}{\mu \pi^{2}} \sum_{p=1}^{\infty} \sum_{q=1}^{\infty} \sum_{n=0}^{\infty} \sum_{m=0}^{\infty} \frac{\mu^{2}}{V_{p} V_{q}} \bar{W}_{t}(p, q, t) \frac{\operatorname{Sin}(2 n+1) \pi c t}{2 n+1} \\
& \cdot \frac{\operatorname{Sin}(2 m+1) \pi y_{0}}{2 m+1} \\
& \times H_{2}^{c}(n, p, j) F^{c}(m, q, k),
\end{aligned}
$$

where

$$
\begin{gathered}
H_{2}^{s}(n, p, j)=\int_{0}^{L_{x}} \operatorname{Sin}(2 n+1) \pi x W_{p}^{\prime \prime}(x) W_{j}(x) d x, \\
H_{2}^{c}(n, p, j)=\int_{0}^{L_{x}} \operatorname{Cos}(2 n+1) \pi x W_{p}^{\prime \prime}(x) W_{j}(x) d x, \\
\frac{M g}{\mu} \int_{0}^{L_{y}} \int_{0}^{L_{x}} H(x-c t) H\left(y-y_{0}\right) W_{j}(x) W_{k}(y) d x d y \\
=\frac{M g L_{x} L_{y}}{\mu \lambda_{j} \lambda_{k}}\left[B_{f}(\lambda, j)+\operatorname{Cos} \frac{\lambda_{j} c t}{L_{x}}-A_{j} \operatorname{Sin} \frac{\lambda_{j} c t}{L_{x}}\right. \\
\left.\quad-B_{j} \operatorname{Cos} h \frac{\lambda_{j} c t}{L_{x}}-C_{j} \operatorname{Sin} h \frac{\lambda_{j} c t}{L_{x}}\right] \\
\quad\left[B_{f}(\lambda, k)+\operatorname{Cos} \frac{\lambda_{k} y_{0}}{L_{y}}-A_{k} \operatorname{Sin} \frac{\lambda_{k} y_{0}}{L_{y}}\right. \\
\left.\quad-B_{k} \operatorname{Cos} h \frac{\lambda_{k} y_{0}}{L_{y}}-C_{k} \operatorname{Sin} h \frac{\lambda_{k} y_{0}}{L_{y}}\right],
\end{gathered}
$$

where

$$
\begin{aligned}
& B_{f}(\lambda, j)=-\operatorname{Cos} \lambda_{j}+A_{j} \operatorname{Sin} \lambda_{j}+B_{j} \operatorname{Cos} h \lambda_{j}+C_{j} \operatorname{Sin} h \lambda_{j}, \\
& B_{f}(\lambda, k)=-\operatorname{Cos} \lambda_{k}+A_{k} \operatorname{Sin} \lambda_{k}+B_{k} \operatorname{Cos} h \lambda_{k}+C_{k} \operatorname{Sin} h \lambda_{k} .
\end{aligned}
$$

Substituting the above expressions in (11) and simplifying, we obtain

$$
\begin{array}{r}
\bar{W}_{t t}(j, k, t)+\Omega_{j, k}^{2} \bar{W}(j, k, t)+\frac{K}{\mu} \bar{W}(j, k, t) \\
-R_{0}\left[\sum_{p=1}^{\infty} \sum_{q=1}^{\infty} \bar{W}_{t t}(p, q, t) H_{2}(p, j) F(q, k)\right.
\end{array}
$$

$$
\begin{array}{r}
\left.+\sum_{p=1}^{\infty} \sum_{q=1}^{\infty} \bar{W}_{t t}(p, q, t) H(p, j) F_{2}(q, k)\right] \\
+\Gamma_{1} L_{x} L_{y}\left\{\left\{\frac{1}{16}\left[\sum_{p=1}^{\infty} \sum_{q=1}^{\infty} F(q, k) H(p, j)\right]\right.\right. \\
+\frac{1}{4 \pi}\left[\sum _ { p = 1 } ^ { \infty } \sum _ { q = 1 } ^ { \infty } \sum _ { n = 0 } ^ { \infty } \left(\frac{\operatorname{Cos}(2 n+1) \pi c t}{2 n+1}\right.\right. \\
\times H^{s}(n, p, j) F(q, k) \\
-\frac{\operatorname{Sin}(2 n+1) \pi c t}{2 n+1} \\
\left.\times H^{c}(n, p, j) F(q, k)\right)
\end{array}
$$$$
+\sum_{p=1}^{\infty} \sum_{q=1}^{\infty} \sum_{m=0}^{\infty}\left(\frac{\operatorname{Cos}(2 m+1) \pi y_{0}}{2 m+1}\right.
$$$$
\times F^{s}(m, q, k) H(p, j)
$$$$
-\frac{\operatorname{Sin}(2 m+1) \pi y_{0}}{2 m+1}
$$$$
\left.\left.\times F^{c}(m, q, k) H(p, j)\right)\right]
$$$$
+\frac{1}{\pi^{2}}\left[\sum_{p=1}^{\infty} \sum_{q=1}^{\infty} \sum_{n=0}^{\infty} \sum_{m=0}^{\infty} \frac{\operatorname{Cos}(2 n+1) \pi c t}{2 n+1}\right.
$$$$
\frac{\operatorname{Cos}(2 m+1) \pi y_{0}}{2 m+1}
$$$$
\times H^{s}(n, p, j) F^{s}(m, q, k)
$$$$
-\sum_{p=1}^{\infty} \sum_{q=1}^{\infty} \sum_{n=0}^{\infty} \sum_{m=0}^{\infty} \frac{\operatorname{Cos}(2 n+1) \pi c t}{2 n+1}
$$$$
\frac{\operatorname{Sin}(2 m+1) \pi y_{0}}{2 m+1}
$$$$
\times H^{s}(n, p, j) F^{c}(m, q, k)
$$$$
-\sum_{p=1}^{\infty} \sum_{q=1}^{\infty} \sum_{n=0}^{\infty} \sum_{m=0}^{\infty} \frac{\operatorname{Sin}(2 n+1) \pi c t}{2 n+1}
$$$$
\frac{\operatorname{Cos}(2 m+1) \pi y_{0}}{2 m+1}
$$$$
\times H^{c}(n, p, j) F^{s}(m, q, k)
$$$$
+\sum_{p=1}^{\infty} \sum_{q=1}^{\infty} \sum_{n=0}^{\infty} \sum_{m=0}^{\infty} \frac{\operatorname{Sin}(2 n+1) \pi c t}{2 n+1}
$$$$
\frac{\operatorname{Sin}(2 m+1) \pi y_{0}}{2 m+1}
$$$$
\times H^{c}(n, p, j)
$$ 


$$
\left.\left.\times F^{c}(m, q, k)\right]\right\}
$$

$\times \bar{W}_{t t}(p, q, t)$

$$
+2 c\left\{\frac{1}{16}\left[\sum_{p=1}^{\infty} \sum_{q=1}^{\infty} H_{1}(p, j) F(q, k)\right]+\frac{1}{4 \pi}\right.
$$$$
\times\left[\sum _ { p = 1 } ^ { \infty } \sum _ { q = 1 } ^ { \infty } \sum _ { n = 0 } ^ { \infty } \left(\frac{\operatorname{Cos}(2 n+1) \pi c t}{2 n+1}\right.\right.
$$$$
\times H_{1}^{s}(n, p, j) F(q, k)
$$$$
-\frac{\operatorname{Sin}(2 n+1) \pi c t}{2 n+1}
$$$$
\left.\times H_{1}^{c}(n, p, j) F(q, k)\right)
$$$$
+\sum_{p=1}^{\infty} \sum_{q=1}^{\infty} \sum_{m=0}^{\infty}\left(\frac{\operatorname{Cos}(2 m+1) \pi y_{0}}{2 m+1}\right.
$$$$
\times F^{s}(m, q, k) H_{1}(p, j)
$$$$
-\frac{\operatorname{Sin}(2 m+1) \pi y_{0}}{2 m+1}
$$$$
\left.\left.\times F^{c}(m, q, k) H_{1}(p, j)\right)\right]
$$

$$
\begin{aligned}
+\frac{1}{\pi^{2}}\left[\sum_{p=1}^{\infty} \sum_{q=1}^{\infty} \sum_{n=0}^{\infty} \sum_{m=0}^{\infty}\right. & \frac{\operatorname{Cos}(2 n+1) \pi c t}{2 n+1} \\
& \frac{\operatorname{Cos}(2 m+1) \pi y_{0}}{2 m+1} \\
& \times H_{1}^{s}(n, p, j) F^{s}(m, q, k)
\end{aligned}
$$

$-\sum_{p=1}^{\infty} \sum_{q=1}^{\infty} \sum_{n=0}^{\infty} \sum_{m=0}^{\infty} \frac{\operatorname{Cos}(2 n+1) \pi c t}{2 n+1}$

$$
\begin{aligned}
& \cdot \frac{\operatorname{Sin}(2 m+1) \pi y_{0}}{2 m+1} \\
& \times H_{1}^{s}(n, p, j) F^{c}(m, q, k)
\end{aligned}
$$

$-\sum_{p=1}^{\infty} \sum_{q=1}^{\infty} \sum_{n=0}^{\infty} \sum_{m=0}^{\infty} \frac{\operatorname{Sin}(2 n+1) \pi c t}{2 n+1}$

$$
\begin{aligned}
& \cdot \frac{\operatorname{Cos}(2 m+1) \pi y_{0}}{2 m+1} \\
& \times H_{1}^{c}(n, p, j) F^{s}(m, q, k)
\end{aligned}
$$

$+\sum_{p=1}^{\infty} \sum_{q=1}^{\infty} \sum_{n=0}^{\infty} \sum_{m=0}^{\infty} \frac{\operatorname{Sin}(2 n+1) \pi c t}{2 n+1}$

$$
\frac{\operatorname{Sin}(2 m+1) \pi y_{0}}{2 m+1}
$$

$$
\left.\left.\times H_{1}^{c}(n, p, j) F^{c}(m, q, k)\right]\right\}
$$

$$
\begin{gathered}
\times \bar{W}_{t}(p, q, t) \\
+c^{2}\left\{\frac{1}{16}\left[\sum_{p=1}^{\infty} \sum_{q=1}^{\infty} H_{2}(p, j) F(q, k)\right]\right. \\
+\frac{1}{4 \pi}\left[\sum _ { p = 1 } ^ { \infty } \sum _ { q = 1 } ^ { \infty } \sum _ { n = 0 } ^ { \infty } \left(\frac{\operatorname{Cos}(2 n+1) \pi c t}{2 n+1}\right.\right. \\
\times H_{2}^{s}(n, p, j) F(q, k) \\
+\frac{\operatorname{Sin}(2 n+1) \pi c t}{2 n+1} \\
+\sum_{p=1}^{\infty} \sum_{q=1}^{\infty} \sum_{m=0}^{\infty}\left(\frac{\operatorname{Cos}(2 m+1) \pi y_{0}}{2 m+1}\right. \\
\times F^{s}(m, q, k) H_{2}(p, j) \\
\times \frac{\operatorname{Sin}(2 m+1) \pi y_{0}}{2 m+1} \\
\left.\left.\times F^{c}(m, q, k) H_{2}(p, j)\right)\right]
\end{gathered}
$$

$+\frac{1}{\pi^{2}}\left[\sum_{p=1}^{\infty} \sum_{q=1}^{\infty} \sum_{n=0}^{\infty} \sum_{m=0}^{\infty} \frac{\operatorname{Cos}(2 n+1) \pi c t}{2 n+1}\right.$

$$
\begin{aligned}
& \cdot \frac{\operatorname{Cos}(2 m+1) \pi y_{0}}{2 m+1} \\
& \times H_{2}^{s}(n, p, j) F^{s}(m, q, k)
\end{aligned}
$$

$-\sum_{p=1}^{\infty} \sum_{q=1}^{\infty} \sum_{n=0}^{\infty} \sum_{m=0}^{\infty} \frac{\operatorname{Cos}(2 n+1) \pi c t}{2 n+1}$

$$
\begin{aligned}
& \cdot \frac{\operatorname{Sin}(2 m+1) \pi y_{0}}{2 m+1} \\
& \times H_{2}^{s}(n, p, j) F^{c}(m, q, k)
\end{aligned}
$$

$-\sum_{p=1}^{\infty} \sum_{q=1}^{\infty} \sum_{n=0}^{\infty} \sum_{m=0}^{\infty} \frac{\operatorname{Sin}(2 n+1) \pi c t}{2 n+1}$

$$
\begin{aligned}
& \cdot \frac{\operatorname{Cos}(2 m+1) \pi y_{0}}{2 m+1} \\
& \times H_{2}^{c}(n, p, j) F^{s}(m, q, k)
\end{aligned}
$$

$+\sum_{p=1}^{\infty} \sum_{q=1}^{\infty} \sum_{n=0}^{\infty} \sum_{m=0}^{\infty} \frac{\operatorname{Sin}(2 n+1) \pi c t}{2 n+1}$

$$
\frac{\operatorname{Sin}(2 m+1) \pi y_{0}}{2 m+1}
$$




$$
\begin{aligned}
& \times \bar{W}(p, q, t) \\
= & \frac{M g L_{x} L_{y}}{\mu \lambda_{j} \lambda_{k}} \\
\times & {\left[B_{f}(\lambda, j)+\operatorname{Cos} \frac{\lambda_{j} c t}{L_{x}}-A_{j} \operatorname{Sin} \frac{\lambda_{j} c t}{L_{x}}\right.} \\
& \left.\quad-B_{j} \operatorname{Cosh} \frac{\lambda_{j} c t}{L_{x}}-C_{j} \operatorname{Sin} h \frac{\lambda_{j} c t}{L_{x}}\right] \\
\cdot & {\left[B_{f}(\lambda, k)+\operatorname{Cos} \frac{\lambda_{k} y_{0}}{L_{y}}-A_{k} \operatorname{Sin} \frac{\lambda_{k} y_{0}}{L_{y}}\right.} \\
& \left.\quad-B_{k} \operatorname{Cosh} \frac{\lambda_{k} y_{0}}{L_{y}}-C_{k} \operatorname{Sinh} \frac{\lambda_{k} y_{0}}{L_{y}}\right],
\end{aligned}
$$$$
\left.\left.\left.\times H_{2}^{c}(n, p, j) F^{c}(m, q, k)\right]\right\}\right\}
$$

where

$$
\Gamma_{1}=\frac{M}{\mu L_{x} L_{y}} .
$$

Equation (40) is the generalized transformed coupled nonhomogeneous second order ordinary differential equation describing the flexural vibration of the rectangular plate under the action of uniformly distributed loads travelling at constant velocity. It is now the fundamental equation of our dynamical problem and holds for all arbitrary boundary conditions. The initial conditions are given by

$$
\bar{W}(j, k, 0)=0, \quad \bar{W}_{t}(j, k, 0)=0 .
$$

In what follows, two special cases of (40) are discussed.

\section{Solution of the Transformed Equation}

4.1. Isotropic Rectangular Plate Traversed by Uniformly Distributed Moving Force. An approximate model which assumes the inertia effect of the uniformly distributed moving mass $M$ as negligible is obtained when the mass ratio $\Gamma_{1}$ is set to zero in (40). Thus, setting $\Gamma_{1}=0$ in (40), one obtains

$$
\begin{aligned}
\bar{W}_{t t}(j, k, t)+\Omega_{j, k}^{2} \bar{W}(j, k, t) \\
\quad-R_{0}\left[\sum_{p=1}^{\infty} \sum_{q=1}^{\infty} H_{2}(p, j) F(q, k)+H(p, j) F_{2}(q, k)\right]
\end{aligned}
$$

$$
\begin{gathered}
\times \bar{W}_{t t}(p, q, t)+\frac{K}{\mu} \bar{W}(j, k, t) \\
=\frac{M g L_{x} L_{y}}{\mu \lambda_{j} \lambda_{k}}\left[B_{f}(\lambda, j)+\operatorname{Cos} \frac{\lambda_{j} c t}{L_{x}}-A_{j} \operatorname{Sin} \frac{\lambda_{j} c t}{L_{x}}\right. \\
\left.\quad-B_{j} \operatorname{Cosh} \frac{\lambda_{j} c t}{L_{x}}-C_{j} \operatorname{Sin} h \frac{\lambda_{j} c t}{L_{x}}\right] \\
\cdot\left[B_{f}(\lambda, k)+\operatorname{Cos} \frac{\lambda_{k} y_{0}}{L_{y}}-A_{k} \operatorname{Sin} \frac{\lambda_{k} y_{0}}{L_{y}}\right. \\
\left.\quad-B_{k} \operatorname{Cosh} \frac{\lambda_{k} y_{0}}{L_{y}}-C_{k} \operatorname{Sin} h \frac{\lambda_{k} y_{0}}{L_{y}}\right] .
\end{gathered}
$$

This represents the classical case of a uniformly distributed moving force problem associated with our dynamical system. Evidently, an analytical solution to (43) is not possible. Consequently, we resort to the modified asymptotic technique due to Struble discussed in Nayfeh [24]. By this technique, we seek the modified frequency corresponding to the frequency of the free system due to the presence of the effect of the rotatory inertia. An equivalent free system operator defined by the modified frequency then replaces (43). To this end, (43) is rearranged to take the form

$$
\begin{aligned}
\bar{W}_{t t} & (j, k, t) \\
+ & \left(\frac{\Omega_{m f}^{* 2}}{\left(1-\varepsilon_{1} L_{x} L_{y}\left[H_{2}(j, j) F(k, k)+H(j, j) F_{2}(k, k)\right]\right)}\right) \\
\times & \bar{W}(j, k, t) \\
- & \frac{\varepsilon_{1}}{\left(1-\varepsilon_{1} L_{x} L_{y}\left[H_{2}(j, j) F(k, k)+H(j, j) F_{2}(k, k)\right]\right)} \\
\times & \sum_{p=1}^{\infty} \sum_{q=1}^{\infty} L_{x} L_{y}\left\{H_{2}(p, j) F(q, k)+H(p, j) F_{2}(q, k)\right\} \\
& \cdot \bar{W}_{t t}(p, q, t) \\
= & \frac{P_{1}}{\left(1-\varepsilon_{1} L_{x} L_{y}\left[H_{2}(j, j) F(k, k)+H(j, j) F_{2}(k, k)\right]\right)} \\
& \cdot\left[B_{f}(\lambda, j)+\operatorname{Cos} \frac{\lambda_{j} c t}{L_{x}}-A_{j} \operatorname{Sin} \frac{\lambda_{j} c t}{L_{x}}\right. \\
& \left.\quad-B_{j} \operatorname{Cos} h \frac{\lambda_{j} c t}{L_{x}}-C_{j} \operatorname{Sin} h \frac{\lambda_{j} c t}{L_{x}}\right],
\end{aligned}
$$


where

$$
\begin{gathered}
\Omega_{m f}^{* 2}=\left(\Omega_{j, k}^{2}+\frac{K}{\mu}\right), \quad \varepsilon_{1}=\frac{R_{0}}{L_{x} L_{y}}, \\
P_{1}=\frac{M g L_{x} L_{y}}{\mu \lambda_{j} \lambda_{k}} W\left(\lambda_{k}, y_{0}\right), \\
W\left(\lambda_{k}, y_{0}\right) \\
=B_{f}(\lambda, k)+\operatorname{Cos} \frac{\lambda_{k} y_{0}}{L_{y}}-A_{k} \operatorname{Sin} \frac{\lambda_{k} y_{0}}{L_{y}} \\
-B_{k} \operatorname{Cos} h \frac{\lambda_{k} y_{0}}{L_{y}}-C_{k} \operatorname{Sin} h \frac{\lambda_{k} y_{0}}{L_{y}} .
\end{gathered}
$$

We now set the right hand side of (44) to zero and consider a parameter $\eta<1$, for any arbitrary ratio $\varepsilon_{1}$, defined as

$$
\eta=\frac{\varepsilon_{1}}{1+\varepsilon_{1}}
$$

Then,

$$
\varepsilon_{1}=\eta+o\left(\eta^{2}\right)
$$

And, consequently, we have that

$$
\begin{aligned}
& \frac{1}{\left(1-\eta L_{x} L_{y}\left[H_{2}(j, j) F(k, k)+H(j, j) F_{2}(k, k)\right]\right)} \\
& =1+\eta L_{x} L_{y}\left[H_{2}(j, j) F(k, k)+H(j, j) F_{2}(k, k)\right] \\
& +o\left(\eta^{2}\right)
\end{aligned}
$$

where

$$
\left|\eta L_{x} L_{y}\left[H_{2}(j, j) F(k, k)+H(j, j) F_{2}(k, k)\right]\right|<1 .
$$

Substituting (47) and (48) into the homogeneous part of (44) one obtains

$$
\begin{aligned}
& \bar{W}_{t t}(j, k, t) \\
& +\left(\Omega_{m f}^{* 2}+\eta \Omega_{m f}^{* 2} L_{x} L_{y}\right. \\
& \left.\times\left[H_{2}(j, j) F(k, k)+H(j, j) F_{2}(k, k)\right]\right) \bar{W}(j, k, t) \\
& -\eta\left\{\sum_{\substack{p=1 \\
p \neq j}}^{\infty} \sum_{\substack{q \neq 1 \\
q \neq k}}^{\infty} L_{x} L_{y}\left[H_{2}(j, j) F(k, k)+H(j, j) F_{2}(k, k)\right]\right\} \\
& \times \bar{W}_{t t}(p, q, t)=0 .
\end{aligned}
$$

When $\eta$ is set to zero in (50) one obtains a situation corresponding to the case in which the rotatory inertia effect on the vibrations of the plate is regarded as negligible. In such a case, the solution of (50) can be obtained as

$$
\bar{W}(j, k, t)=C_{m f} \operatorname{Cos}\left[\Omega_{m f}^{*} t-\phi_{m f}\right],
$$

where $C_{m f}$ and $\phi_{m f}$ are constants.
Since $\eta<1$, Struble's technique requires that the solution to (50) be of the form [24]

$$
\begin{aligned}
\bar{W}(j, k, t)= & A(j, k, t) \operatorname{Cos}\left[\Omega_{m f}^{*} t-\phi(j, k, t)\right] \\
& +\eta \bar{W}_{1}(j, k, t)+o\left(\eta^{2}\right),
\end{aligned}
$$

where $A(j, k, t)$ and $\phi(j, k, t)$ are slowly varying functions of time. To obtain the modified frequency, (52) and its derivatives are substituted into (50). After some simplifications and rearrangements, one obtains

$$
\begin{aligned}
& {\left[2 \Omega_{m f}^{*} \dot{\phi}(j, k, t)+\eta \Omega_{m f}^{* 2} L_{x} L_{y}\right.} \\
& \left.\times\left[H_{2}(j, j) F(k, k)+H(j, j) F_{2}(k, k)\right]\right] \\
& \times A(j, k, t) \operatorname{Cos}\left[\Omega_{m f}^{*} t-\phi(j, k, t)\right] \\
& -2 \dot{A}(j, k, t) \Omega_{m f}^{*} \operatorname{Sin}\left[\Omega_{m f}^{*} t-\phi(j, k, t)\right] \\
& -\eta \sum_{p=1}^{\infty} \sum_{q=1}^{\infty}\left\{L_{x} L_{y}\left\{H_{2}(j, j) F(k, k)+H(j, j) F_{2}(k, k)\right\}\right. \\
& \cdot\left\{2 \Omega_{m f}^{*} \dot{\phi}(p, q, t)-\Omega_{m f}^{* 2}\right\} A(p, q, t) \\
& \quad \times \operatorname{Cos}\left[\Omega_{m f}^{*} t-\phi(j, k, t)\right] \\
& \left.-2 \dot{A}(p, q, t) \Omega_{m f}^{*} \operatorname{Sin}\left[\Omega_{m f}^{*} t-\phi(p, q, t)\right]\right\}
\end{aligned}
$$

neglecting terms to $o\left(\eta^{2}\right)$.

The variational equations are obtained by equating the coefficients of $\operatorname{Sin}\left[\Omega_{m f}^{*} t-\phi(j, k, t)\right]$ and $\operatorname{Cos}\left[\Omega_{m f}^{*} t-\phi(j, k, t)\right]$ on both sides of (53) to zero; thus, one obtains

$$
\begin{gathered}
-2 \dot{A}(j, k, t) \Omega_{m f}^{*}=0, \\
{\left[2 \Omega_{m f}^{*} \dot{\phi}(j, k, t)+\eta \Omega_{m f}^{* 2} L_{x} L_{y}\right.} \\
\left.\times\left[H_{2}(j, j) F(k, k)+H(j, j) F_{2}(k, k)\right]\right]=0 .
\end{gathered}
$$

Evaluating (54) one obtains

$$
\begin{gathered}
A(j, k, t)=A_{j k}, \\
\phi(j, k, t)=-\eta \Omega_{m f}^{*} \frac{L_{x} L_{y}}{2} \\
\times\left[H_{2}(j, j) F(k, k)+H(j, j) F_{2}(k, k)\right] t+\phi_{f},
\end{gathered}
$$

where $A_{j k}$ and $\phi_{f}$ are constants.

Therefore, when the effect of the rotatory inertia correction factor is considered, the first approximation to the homogeneous system is given by

$$
V(j, k, t)=A_{j k} \operatorname{Cos}\left[\gamma_{p m f} t-\phi_{f}\right],
$$


where

$$
\begin{aligned}
\gamma_{p m f}= & \Omega_{m f}^{*} \\
& \times\left\{1+\frac{\eta}{2}\left(L_{x} L_{y}\left[H_{2}(j, j) F(k, k)+H(j, j) F_{2}(k, k)\right]\right)\right\}
\end{aligned}
$$

represents the modified natural frequency due to the presence of rotatory inertia correction factor. It is observed that when $\eta=0$, we recover the frequency of the moving force problem when the rotatory inertia effect of the plate is neglected. Thus, to solve the nonhomogeneous equation (44), the differential operator which acts on $\bar{W}(j, k, t)$ and $\bar{W}(p, q, t)$ is now replaced by the equivalent free system operator defined by the modified frequency $\gamma_{p m f}$; that is,

$$
\begin{aligned}
\bar{W}_{t t}(j, k, t)+\gamma_{p m f}^{2} \bar{W}(j, k, t) \\
=P_{p m f}\left[B_{f}(\lambda, j)+\operatorname{Cos} \frac{\lambda_{j} c t}{L_{x}}-A_{j} \operatorname{Sin} \frac{\lambda_{j} c t}{L_{x}}\right. \\
\left.-B_{j} \operatorname{Cos} h \frac{\lambda_{j} c t}{L_{x}}-C_{j} \operatorname{Sin} h \frac{\lambda_{j} c t}{L_{x}}\right],
\end{aligned}
$$

where

$$
P_{p m f}=\frac{P_{1}}{\left(1-\eta L_{x} L_{y}\left[H_{2}(j, j) F(k, k)+H(j, j) F_{2}(k, k)\right]\right)} .
$$

To obtain the solution to (58), it is subjected to a Laplace transform. Thus, solving (58) in conjunction with the initial condition, the solution is given by

$$
\begin{gathered}
\bar{V}(j, k, t) \\
=P_{p m f}\left[\frac{B_{f}(\lambda, j)\left(1-\operatorname{Cos} \gamma_{p m f} t\right)}{\gamma_{p m f}}+\frac{\operatorname{Cos} \alpha_{j} t-\operatorname{Cos} \gamma_{p m f} t}{\gamma_{p m f}^{2}-\alpha_{j}^{2}}\right. \\
-\frac{A_{j}\left(\gamma_{p m f} \operatorname{Sin} \alpha_{j} t-\alpha_{j} \operatorname{Sin} \gamma_{p m f} t\right)}{\gamma_{p m f}\left(\gamma_{p m f}^{2}-\alpha_{j}^{2}\right)} \\
-\frac{B_{j}\left(\operatorname{Cos} h \alpha_{j} t-\operatorname{Cos} \gamma_{p m f} t\right)}{\alpha_{j}^{2}+\gamma_{p m f}^{2}} \\
\left.-\frac{C_{j}\left(\gamma_{p m f} \operatorname{Sin} h \alpha_{j} t-\alpha_{j} \operatorname{Sin} \gamma_{p m f} t\right)}{\gamma_{p m f}\left(\alpha_{c}^{2}+\gamma_{p m f}^{2}\right)}\right],
\end{gathered}
$$

where

$$
\alpha_{j}=\frac{\lambda_{j} c}{L_{x}} .
$$

Substituting (60) into (8), we have

$$
\begin{aligned}
& W(x, y, t) \\
& =\sum_{j=1}^{\infty} \sum_{k=1}^{\infty} \frac{1}{V_{j} V_{k}} P_{p m f}\left[\frac{B_{f}(\lambda, j)\left(1-\operatorname{Cos} \gamma_{p m f} t\right)}{\gamma_{p m f}}\right. \\
& +\frac{\operatorname{Cos} \alpha_{j} t-\operatorname{Cos} \gamma_{p m f} t}{\gamma_{p m f}^{2}-\alpha_{j}^{2}} \\
& -\frac{A_{j}\left(\gamma_{p m f} \operatorname{Sin} \alpha_{j} t-\alpha_{j} \operatorname{Sin} \gamma_{p m f} t\right)}{\gamma_{p m f}\left(\gamma_{p m f}^{2}-\alpha_{j}^{2}\right)} \\
& -\frac{B_{j}\left(\operatorname{Cosh} \alpha_{j} t-\operatorname{Cos} \gamma_{p m f} t\right)}{\alpha_{j}^{2}+\gamma_{p m f}^{2}} \\
& \left.-\frac{C_{j}\left(\gamma_{p m f} \operatorname{Sin} h \alpha_{j} t-\alpha_{j} \operatorname{Sin} \gamma_{p m f} t\right)}{\gamma_{p m f}\left(\alpha_{j}^{2}+\gamma_{p m f}^{2}\right)}\right] \\
& \cdot\left(\operatorname{Sin} \frac{\lambda_{j} x}{L_{x}}+A_{j} \operatorname{Cos} \frac{\lambda_{j} x}{L_{x}}+B_{j} \operatorname{Sin} h \frac{\lambda_{j} x}{L_{x}}+C_{j} \operatorname{Cosh} \frac{\lambda_{j} x}{L_{x}}\right) \\
& \cdot\left(\operatorname{Sin} \frac{\lambda_{k} y}{L_{y}}+A_{k} \operatorname{Cos} \frac{\lambda_{k} y}{L_{y}}+B_{k} \operatorname{Sin} h \frac{\lambda_{k} y}{L_{y}}+C_{k} \operatorname{Cosh} \frac{\lambda_{k} y}{L_{y}}\right) .
\end{aligned}
$$

Equation (62) represents the transverse displacement response to a uniformly distributed force moving at constant velocity for arbitrary boundary conditions of a rectangular plate incorporating the effects of rotatory inertia correction factor and resting on elastic foundation.

4.2. Isotropic Rectangular Plate Traversed by Uniformly Distributed Moving Mass. In this section, we consider the case in which the mass of the structure and that of the load are of comparable magnitude. Under such condition, the inertia effect of the uniformly distributed moving mass is not negligible. Thus, $\Gamma_{1} \neq 0$, and the solution to the entire equation (40) is required. This gives the moving mass problem. An exact analytical solution to this dynamical problem is not possible. Again, we resort to the modified asymptotic technique due to Struble discussed in Nayfeh [24]. Evidently, the homogenous part of (40) can be replaced by a free system operator defined by the modified frequency due to the presence of rotatory inertia correction factor $R_{0}$. To this end, (40) can now be rearranged to take the form

$$
\begin{gathered}
\bar{W}_{t t}(j, k, t)+\frac{2 c \Gamma_{1} L_{x} L_{y} G_{2}(j, k, t)}{1+\Gamma_{1} L_{x} L_{y} G_{1}(j, k, t)} \bar{W}_{t}(j, k, t) \\
+\frac{c^{2} \Gamma_{1} L_{x} L_{y} G_{3}(j, k, t)+\gamma_{p m f}^{2}}{1+\Gamma_{1} L_{x} L_{y} G_{1}(j, k, t)} \bar{W}(j, k, t)
\end{gathered}
$$




$$
\begin{gathered}
+\frac{\Gamma_{1} L_{x} L_{y}}{1+\Gamma_{1} L_{x} L_{y} G_{1}(j, k, t)} \\
\times \sum_{\substack{p=1 \\
p \neq j}}^{\infty} \sum_{\substack{q=1 \\
q \neq k}}^{\infty}\left\{G_{a}(p, q, t) \bar{W}_{t t}(p, q, t)+2 c G_{b}(p, q, t)\right. \\
\left.\times \bar{W}_{t}(p, q, t)+c^{2} G_{c}(p, q, t) \bar{W}(p, q, t)\right\} \\
=\frac{M g L_{x} L_{y} W\left(\lambda_{k}, y_{0}\right)}{\mu \lambda_{j} \lambda_{k}\left[1+\Gamma_{1} L_{x} L_{y} G_{1}(j, k, t)\right]} \\
\times\left[B_{f}(\lambda, j)+\operatorname{Cos} \frac{\lambda_{j} c t}{L_{x}}-A_{j} \operatorname{Sin} \frac{\lambda_{j} c t}{L_{x}}\right. \\
\left.-B_{j} \operatorname{Cos} h \frac{\lambda_{j} c t}{L_{x}}-C_{j} \operatorname{Sin} h \frac{\lambda_{j} c t}{L_{x}}\right],
\end{gathered}
$$

where

$$
\begin{aligned}
& G_{1}(j, k, t) \\
& =\frac{1}{16} F(k, k) H(j, j) \\
& +\frac{1}{4 \pi}\left[\sum _ { n = 0 } ^ { \infty } \left(\frac{\operatorname{Cos}(2 n+1) \pi c t}{2 n+1} H^{s}(n, j, j) F(k, k)\right.\right. \\
& \left.-\frac{\operatorname{Sin}(2 n+1) \pi c t}{2 n+1} H^{c}(n, j, j) F(k, k)\right) \\
& +\sum_{m=0}^{\infty}\left(\frac{\operatorname{Cos}(2 m+1) \pi y_{0}}{2 m+1} F^{s}(m, k, k) H(j, j)\right. \\
& \left.\left.-\frac{\operatorname{Sin}(2 m+1) \pi y_{0}}{2 m+1} F^{c}(m, k, k) H(j, j)\right)\right] \\
& -\frac{1}{\pi^{2}}\left[\sum_{n=0}^{\infty} \sum_{m=0}^{\infty} \frac{\operatorname{Cos}(2 n+1) \pi c t}{2 n+1} \cdot \frac{\operatorname{Sin}(2 m+1) \pi y_{0}}{2 m+1}\right. \\
& \times H^{s}(n, j, j) F^{c}(m, k, k) \\
& -\sum_{n=0}^{\infty} \sum_{m=0}^{\infty} \frac{\operatorname{Sin}(2 n+1) \pi c t}{2 n+1} \cdot \frac{\operatorname{Cos}(2 m+1) \pi y_{0}}{2 m+1} \\
& \times H^{c}(n, j, j) F^{s}(m, k, k) \\
& +\sum_{n=0}^{\infty} \sum_{m=0}^{\infty} \frac{\operatorname{Sin}(2 n+1) \pi c t}{2 n+1} \cdot \frac{\operatorname{Sin}(2 m+1) \pi y_{0}}{2 m+1} \\
& \left.\times H^{c}(n, p, j) F^{c}(m, q, k)\right] \\
& G_{2}(j, k, t) \\
& =\frac{1}{16} F(k, k) H_{1}(j, j)
\end{aligned}
$$

$$
\begin{array}{r}
+\frac{1}{4 \pi}\left[\sum _ { n = 0 } ^ { \infty } \left(\frac{\operatorname{Cos}(2 n+1) \pi c t}{2 n+1} H_{1}^{s}(n, j, j) F(k, k)\right.\right. \\
\left.-\frac{\operatorname{Sin}(2 n+1) \pi c t}{2 n+1} H_{1}^{c}(n, j, j) F(k, k)\right) \\
+\sum_{m=0}^{\infty}\left(\frac{\operatorname{Cos}(2 m+1) \pi y_{0}}{2 m+1} F^{s}(m, k, k) H_{1}(j, j)\right. \\
\left.\left.\quad-\frac{\operatorname{Sin}(2 m+1) \pi y_{0}}{2 m+1} F^{c}(m, k, k) H_{1}(j, j)\right)\right] \\
+\frac{1}{\pi^{2}}\left[\sum_{n=0}^{\infty} \sum_{m=0}^{\infty} \frac{\operatorname{Cos}(2 n+1) \pi c t}{2 n+1} \cdot \frac{\operatorname{Cos}(2 m+1) \pi y_{0}}{2 m+1}\right. \\
\times H_{1}^{s}(n, j, j) F^{s}(m, k, k)
\end{array}
$$$$
-\sum_{n=0}^{\infty} \sum_{m=0}^{\infty} \frac{\operatorname{Cos}(2 n+1) \pi c t}{2 n+1} \cdot \frac{\operatorname{Sin}(2 m+1) \pi y_{0}}{2 m+1}
$$$$
\times H_{1}^{s}(n, j, j) F^{c}(m, k, k)
$$$$
-\sum_{n=0}^{\infty} \sum_{m=0}^{\infty} \frac{\operatorname{Sin}(2 n+1) \pi c t}{2 n+1} \cdot \frac{\operatorname{Cos}(2 m+1) \pi y_{0}}{2 m+1}
$$$$
\times H_{1}^{c}(n, j, j) F^{s}(m, k, k)
$$$$
+\sum_{n=0}^{\infty} \sum_{m=0}^{\infty} \frac{\operatorname{Sin}(2 n+1) \pi c t}{2 n+1} \cdot \frac{\operatorname{Sin}(2 m+1) \pi y_{0}}{2 m+1}
$$$$
\left.\times H_{1}^{c}(n, j, j) F^{c}(m, k, k)\right]
$$

$$
\begin{aligned}
& G_{3}(j, k, t) \\
& =\frac{1}{16} F(k, k) H_{2}(j, j) \\
& +\frac{1}{4 \pi}\left[\sum _ { n = 0 } ^ { \infty } \left(\frac{\operatorname{Cos}(2 n+1) \pi c t}{2 n+1} H_{2}^{s}(n, j, j) F(k, k)\right.\right. \\
& \left.-\frac{\operatorname{Sin}(2 n+1) \pi c t}{2 n+1} H_{2}^{c}(n, j, j) F(k, k)\right) \\
& +\sum_{m=0}^{\infty}\left(\frac{\operatorname{Cos}(2 m+1) \pi y_{0}}{2 m+1} F^{s}(m, k, k) H_{2}(j, j)\right. \\
& \left.\left.-\frac{\operatorname{Sin}(2 m+1) \pi y_{0}}{2 m+1} F^{c}(m, k, k) H_{2}(j, j)\right)\right] \\
& +\frac{1}{\pi^{2}}\left[\sum_{n=0}^{\infty} \sum_{m=0}^{\infty} \frac{\operatorname{Cos}(2 n+1) \pi c t}{2 n+1} \cdot \frac{\operatorname{Cos}(2 m+1) \pi y_{0}}{2 m+1}\right. \\
& \times H_{2}^{s}(n, j, j) F^{s}(m, k, k) \\
& -\sum_{n=0}^{\infty} \sum_{m=0}^{\infty} \frac{\operatorname{Cos}(2 n+1) \pi c t}{2 n+1} \cdot \frac{\operatorname{Sin}(2 m+1) \pi y_{0}}{2 m+1}
\end{aligned}
$$




$$
\begin{aligned}
& \quad \times H_{2}^{s}(n, j, j) F^{c}(m, k, k) \\
& -\sum_{n=0}^{\infty} \sum_{m=0}^{\infty} \frac{\operatorname{Sin}(2 n+1) \pi c t}{2 n+1} \cdot \frac{\operatorname{Cos}(2 m+1) \pi y_{0}}{2 m+1} \\
& \quad \times H_{2}^{c}(n, j, j) F^{s}(m, k, k) \\
& +\sum_{n=0}^{\infty} \sum_{m=0}^{\infty} \frac{\operatorname{Sin}(2 n+1) \pi c t}{2 n+1} \cdot \frac{\operatorname{Sin}(2 m+1) \pi y_{0}}{2 m+1} \\
& \left.\times H_{2}^{c}(n, j, j) F^{c}(m, k, k)\right], \\
& G_{a}(p, q, t)=\left.G_{1}(j, k, t)\right|_{j=p, k=q}, \\
& G_{b}(p, q, t)=\left.G_{2}(j, k, t)\right|_{j=p, k=q^{\prime}} \\
& G_{c}(p, q, t)=\left.G_{3}(j, k, t)\right|_{j=p, k=q} .
\end{aligned}
$$

Going through similar argument as in the previous section, we obtain the first approximation to the homogenous system when the effect of the mass of the uniformly distributed load is considered as

$$
\bar{W}(j, k, t)=\Delta_{j k} e^{-\beta(j, k) t} \operatorname{Cos}\left[\gamma_{m m} t-\phi_{m}\right],
$$

where $\phi_{m}$ and $\Delta_{j k}$ are constants. Consider

$$
\beta(j, k) t=c \Gamma_{a} L_{x} L_{y} H_{c}(j, k), \quad \Gamma_{a}=\frac{\Gamma_{1}}{1+\Gamma_{1}},
$$

where

$$
\gamma_{m m}=\gamma_{p m f}\left\{1-\frac{\Gamma_{a} L_{x} L_{y}}{2}\left[H_{b}(j, k)-\frac{c^{2} H_{a}(j, k)}{\gamma_{p m f}^{2}}\right]\right\}
$$

is called the modified natural frequency representing the frequency of the free system due to the presence of the uniformly distributed moving mass and

$$
\begin{aligned}
& H_{a}(j, k) \\
& \quad=\frac{1}{16} F(k, k) H_{2}(j, j)
\end{aligned}
$$

$$
\begin{aligned}
+\frac{1}{4 \pi}\left[\sum_{n=0}^{\infty}(\right. & \frac{\operatorname{Cos}(2 n+1) \pi c t}{2 n+1} H_{2}^{s}(n, j, j) F(k, k) \\
& \left.-\frac{\operatorname{Sin}(2 n+1) \pi c t}{2 n+1} H_{2}^{c}(n, j, j) F(k, k)\right) \\
+ & \sum_{m=0}^{\infty}\left(\frac{\operatorname{Cos}(2 m+1) \pi y_{0}}{2 m+1} F^{s}(m, k, k) H_{2}(j, j)\right. \\
& \left.\left.-\frac{\operatorname{Sin}(2 m+1) \pi y_{0}}{2 m+1} F^{c}(m, k, k) H_{2}(j, j)\right)\right],
\end{aligned}
$$

$$
\begin{aligned}
& H_{b}(j, k) \\
& =\frac{1}{16} F(k, k) H(j, j) \\
& +\frac{1}{4 \pi}\left[\sum _ { n = 0 } ^ { \infty } \left(\frac{\operatorname{Cos}(2 n+1) \pi c t}{2 n+1} H^{s}(n, j, j) F(k, k)\right.\right. \\
& \left.\quad-\frac{\operatorname{Sin}(2 n+1) \pi c t}{2 n+1} H^{c}(n, j, j) F(k, k)\right) \\
& +\sum_{m=0}^{\infty}\left(\frac{\operatorname{Cos}(2 m+1) \pi y_{0}}{2 m+1} F^{s}(m, k, k) H(j, j)\right. \\
& \left.\left.\quad-\frac{\operatorname{Sin}(2 m+1) \pi y_{0}}{2 m+1} F^{c}(m, k, k) H(j, j)\right)\right],
\end{aligned}
$$

$H_{c}(j, k)$

$$
\begin{aligned}
& =\frac{1}{16} F(k, k) H_{1}(j, j) \\
& +\frac{1}{4 \pi}\left[\sum _ { n = 0 } ^ { \infty } \left(\frac{\operatorname{Cos}(2 n+1) \pi c t}{2 n+1} H_{1}^{s}(n, j, j) F(k, k)\right.\right. \\
& \left.\quad-\frac{\operatorname{Sin}(2 n+1) \pi c t}{2 n+1} H_{1}^{c}(n, j, j) F(k, k)\right) \\
& +\sum_{m=0}^{\infty}\left(\frac{\operatorname{Cos}(2 m+1) \pi y_{0}}{2 m+1} F^{s}(m, k, k) H_{1}(j, j)\right. \\
& \left.\left.-\frac{\operatorname{Sin}(2 m+1) \pi y_{0}}{2 m+1} F^{c}(m, k, k) H_{1}(j, j)\right)\right] .
\end{aligned}
$$

To solve the nonhomogeneous equation (63), the differential operator which acts on $\bar{W}(j, k, t)$ and $\bar{W}(p, q, t)$ is replaced by the equivalent free system operator defined by the modified frequency $\gamma_{m m}$; that is,

$$
\begin{gathered}
\bar{W}_{t t}(j, k, t)+\gamma_{m m}^{2} \bar{W}(j, k, t) \\
=\frac{\Gamma_{a} g L_{x}^{2} L_{y}^{2} W\left(\lambda_{k}, y_{0}\right)}{\lambda_{j} \lambda_{k}}
\end{gathered}
$$




$$
\begin{aligned}
& \times\left[B_{f}(\lambda, j)+\operatorname{Cos} \frac{\lambda_{j} c t}{L_{x}}\right. \\
& \left.\quad-A_{j} \operatorname{Sin} \frac{\lambda_{j} c t}{L_{x}}-B_{j} \operatorname{Cosh} \frac{\lambda_{j} c t}{L_{x}}-C_{j} \operatorname{Sin} h \frac{\lambda_{j} c t}{L_{x}}\right] .
\end{aligned}
$$

Evidently, (69) is analogous to (58). Thus, using similar argument as in the previous section, solution to (69) can be obtained as

$$
\begin{aligned}
& W(x, y, t) \\
& =\sum_{j=1}^{\infty} \sum_{k=1}^{\infty} \frac{1}{V_{j} V_{k}} \frac{\Gamma_{a} g L_{x}^{2} L_{y}^{2} W\left(\lambda_{k}, y_{0}\right)}{\lambda_{j} \lambda_{k}} \\
& \times\left[\frac{B_{f}(\lambda, j)\left(1-\operatorname{Cos} \gamma_{m m} t\right)}{\gamma_{m m}}\right. \\
& +\frac{\operatorname{Cos} \alpha_{j} t-\operatorname{Cos} \gamma_{m m} t}{\gamma_{m m}^{2}-\alpha_{j}^{2}} \\
& -\frac{A_{j}\left(\gamma_{m m} \operatorname{Sin} \alpha_{j} t-\alpha_{j} \operatorname{Sin} \gamma_{m m} t\right)}{\gamma_{m m}\left(\gamma_{m m}^{2}-\alpha_{j}^{2}\right)} \\
& -\frac{B_{j}\left(\operatorname{Cos} h \alpha_{j} t-\operatorname{Cos} \gamma_{m m} t\right)}{\alpha_{j}^{2}+\gamma_{m m}^{2}} \\
& \left.-\frac{C_{j}\left(\gamma_{m m} \operatorname{Sin} h \alpha_{j} t-\alpha_{j} \operatorname{Sin} \gamma_{m m} t\right)}{\gamma_{m m}\left(\alpha_{j}^{2}+\gamma_{m m}^{2}\right)}\right] \\
& \cdot\left(\operatorname{Sin} \frac{\lambda_{j} x}{L_{x}}+A_{j} \operatorname{Cos} \frac{\lambda_{j} x}{L_{x}}+B_{j} \operatorname{Sin} h \frac{\lambda_{j} x}{L_{x}}+C_{j} \operatorname{Cosh} \frac{\lambda_{j} x}{L_{x}}\right) \\
& \cdot\left(\operatorname{Sin} \frac{\lambda_{k} y}{L_{y}}+A_{k} \operatorname{Cos} \frac{\lambda_{k} y}{L_{y}}+B_{k} \operatorname{Sin} h \frac{\lambda_{k} y}{L_{y}}+C_{k} \operatorname{Cosh} \frac{\lambda_{k} y}{L_{y}}\right) .
\end{aligned}
$$

Equation (70) represents the transverse displacement response to uniformly distributed masses moving at constant velocity of an isotropic rectangular plate resting on elastic foundation for various end conditions.

\section{Illustrative Examples}

In this section, practical examples of classical boundary conditions are selected to illustrate the analyses presented in this paper.

5.1. Rectangular Plate Clamped at Edge $x=0, x=L_{x}$ with Simple Supports at $y=0, y=L_{y}$. In this example, the rectangular plate has simple supports at the edges $y=0$,
$y=L_{y}$ and is clamped at edges $x=0, x=L_{x}$. The boundary conditions at such opposite edges are

$$
\begin{array}{rlrl}
W(0, y, t) & =0, & W\left(L_{x}, y, t\right) & =0, \\
W(x, 0, t) & =0, & W\left(x, L_{y}, t\right)=0, \\
\frac{\partial W(0, y, t)}{\partial x} & =0, & & \frac{\partial W\left(L_{x}, y, t\right)}{\partial x}=0, \\
\frac{\partial^{2} W(x, 0, t)}{\partial y^{2}} & =0, & \frac{\partial^{2} W\left(x, L_{y}, t\right)}{\partial y^{2}} & =0
\end{array}
$$

and hence for normal modes

$$
\begin{array}{rlrl}
W_{j}(0) & =0, & W_{j}\left(L_{x}\right)=0, \\
W_{k}(0) & =0, & W_{k}\left(L_{y}\right)=0, \\
\frac{\partial W_{j}(0)}{\partial x}=0, & \frac{\partial W_{j}\left(L_{x}\right)}{\partial x}=0, \\
\frac{\partial^{2} W_{k}(0)}{\partial y^{2}}=0, & \frac{\partial^{2} W_{k}\left(L_{y}\right)}{\partial y^{2}}=0 .
\end{array}
$$

Using the boundary conditions (71) in (10) the following values of the constants are obtained for the clamped edges:

$$
\begin{gathered}
A_{j}=\frac{\operatorname{Sin} h \lambda_{j}-\operatorname{Sin} \lambda_{j}}{\operatorname{Cos} \lambda_{j}-\operatorname{Cos} h \lambda_{j}} \Longrightarrow A_{p}=\frac{\operatorname{Sin} h \lambda_{p}-\operatorname{Sin} \lambda_{p}}{\operatorname{Cos} \lambda_{p}-\operatorname{Cos} h \lambda_{p}} \\
B_{j}=-1 \Longrightarrow B_{p}=-1 \\
C_{j}=-A_{j} \Longrightarrow C_{p}=-A_{p}
\end{gathered}
$$

The frequency equation for the clamped edges is given by

$$
\operatorname{Cos} \lambda_{j} \operatorname{Cos} h \lambda_{j}-1=0
$$


such that

$$
\begin{aligned}
& \lambda_{1}=4.73004, \quad \lambda_{2}=7.85320, \quad \lambda_{3}=10.99561, \\
& V_{j}=\frac{\mu L_{x}}{2} \\
& \times\left\{1+A_{j}^{2}+B_{j}^{2}+C_{j}^{2}+\frac{1}{\lambda_{j}}\right. \\
& \times\left[2 C_{j}-2 A_{j} B_{j}-B_{j} C_{j}-\frac{1}{2}\left(1-A_{j}^{2}\right) \operatorname{Sin} 2 \lambda_{j}\right. \\
& +\left(B_{j}^{2}+C_{j}^{2}\right) \operatorname{Sin} h \lambda_{j} \cdot \operatorname{Cos} h \lambda_{j}+2 A_{j} \operatorname{Sin}^{2} \lambda_{j} \\
& +2\left(B_{j}+A_{j} C_{j}\right) \operatorname{Sin} \lambda_{j} \operatorname{Cos} h \lambda_{j} \\
& +2\left(-B_{j}+A_{j} C_{j}\right) \operatorname{Sin} h \lambda_{j} \operatorname{Cos} \lambda_{j} \\
& +2\left(C_{j}+A_{j} B_{j}\right) \operatorname{Sin} h \lambda_{j} \operatorname{Sin} \lambda_{j} \\
& +2\left(-C_{j}+A_{j} B_{j}\right) \operatorname{Cos} \lambda_{j} \operatorname{Cos} h \lambda_{j} \\
& \left.\left.+B_{j} C_{j} \operatorname{Cos} h 2 \lambda_{j}\right]\right\} \text {. }
\end{aligned}
$$

$V_{p}$ is obtained by replacing subscript $j$ with $p$ in (77). For the simple edges, it is readily shown that

$$
\begin{gathered}
A_{k}=0 \Longrightarrow A_{q}=0, \quad B_{k}=0 \Longrightarrow B_{q}=0, \\
C_{k}=0 \Longrightarrow C_{q}=0 .
\end{gathered}
$$

The corresponding frequency equation is

$$
\begin{gathered}
\lambda_{k}=k \pi \Longrightarrow \lambda_{q}=q \pi, \\
V_{k}=\frac{\mu L_{y}}{2}, \quad V_{q}=\frac{\mu L_{y}}{2} .
\end{gathered}
$$

Thus, the general solutions of the associated uniformly distributed moving force and uniformly distributed moving mass problems of the simple-clamped rectangular plate are obtained by substituting the above results in (73) to (80) into (62) and (70).

5.2. Rectangular Plate Clamped at All Edges. For a rectangular plate clamped at all edges, both the deflection and the slope vanish at such ends. Thus, the following boundary conditions pertain:

$$
\begin{array}{rlrl}
W(0, y, t) & =0, & W\left(L_{x}, y, t\right)=0, \\
W(x, 0, t)=0, & W\left(x, L_{y}, t\right)=0, \\
\frac{\partial W(0, y, t)}{\partial x}=0, & \frac{\partial W\left(L_{x}, y, t\right)}{\partial x}=0, \\
\frac{\partial W(x, 0, t)}{\partial y}=0, & \frac{\partial W\left(x, L_{y}, t\right)}{\partial y}=0
\end{array}
$$

and hence for normal modes we have

$$
\begin{aligned}
W_{j}(0) & =0, & & W_{j}\left(L_{x}\right)=0, \\
W_{k}(0) & =0, & & W_{k}\left(L_{y}\right)=0, \\
\frac{\partial W_{j}(0)}{\partial x} & =0, & & \frac{\partial W_{j}\left(L_{x}\right)}{\partial x}=0, \\
\frac{\partial W_{k}(0)}{\partial y} & =0, & & \frac{\partial W_{k}\left(L_{y}\right)}{\partial y}=0 .
\end{aligned}
$$

Using the boundary conditions (81)-(82) in (10) one obtains the following values of the constants for the clamped edges at $x=0$ and $x=L_{x}$ :

$$
\begin{gathered}
A_{j}=\frac{\operatorname{Sin} h \lambda_{j}-\operatorname{Sin} \lambda_{j}}{\operatorname{Cos} \lambda_{j}-\operatorname{Cos} h \lambda_{j}} \Longrightarrow A_{p}=\frac{\operatorname{Sin} h \lambda_{p}-\operatorname{Sin} \lambda_{p}}{\operatorname{Cos} \lambda_{p}-\operatorname{Cos} h \lambda_{p}} \\
B_{j}=-1 \Longrightarrow B_{p}=-1, \quad C_{j}=-A_{j} \Longrightarrow C_{p}=-A_{p} .
\end{gathered}
$$

The frequency equation of the clamped edges is given by

$$
\operatorname{Cos} \lambda_{j} \operatorname{Cos} h \lambda_{j}-1=0
$$

such that

$$
\lambda_{1}=4.73004, \quad \lambda_{2}=7.85320, \quad \lambda_{3}=10.99561 .
$$

$V_{j}$ is defined by (77) and $V_{p}$ is obtained by replacing subscript $j$ with $p$.

For the clamped edges at $y=0$ and $y=L_{y}$, the same process is followed to obtain

$$
\begin{aligned}
A_{k} & =\frac{\operatorname{Sin} h \lambda_{k}-\operatorname{Sin} \lambda_{k}}{\operatorname{Cos} \lambda_{k}-\operatorname{Cos} h \lambda_{k}} \Longrightarrow A_{q}=\frac{\operatorname{Sin} h \lambda_{q}-\operatorname{Sin} \lambda_{q}}{\operatorname{Cos} \lambda_{q}-\operatorname{Cos} h \lambda_{q}}, \\
B_{k} & =-1 \Longrightarrow B_{q}=-1, \quad C_{k}=-A_{k} \Longrightarrow C_{q}=-A_{q} .
\end{aligned}
$$

The frequency equation of the clamped edges is given by

$$
\operatorname{Cos} \lambda_{k} \operatorname{Cos} h \lambda_{k}-1=0
$$

such that

$$
\lambda_{1}=4.73004, \quad \lambda_{2}=7.85320, \quad \lambda_{3}=10.99561 .
$$

$V_{k}$ and $V_{q}$ are obtained by replacing subscript $j$ with $k$ and $q$ in (77), respectively.

Thus, the general solutions of the associated uniformly distributed moving force and uniformly distributed moving mass problems of the clamped-clamped rectangular plate are obtained by substituting the above results in (83) to (89) into (62) and (70).

\section{Discussion of the Analytical Solutions}

When an undamped system such as this is studied, one is interested in the resonance conditions of the vibrating system, 
because the transverse displacement of the elastic rectangular plate may increase without bound. Thus, for both illustrative examples, we observe that the isotropic rectangular plate traversed by a uniformly distributed moving force reaches a state of resonance whenever

$$
\gamma_{p m f}=\frac{\lambda_{j} c}{L_{x}}
$$

while the same plate under the action of a uniformly distributed moving mass experiences resonance effect whenever

$$
\gamma_{m m}=\frac{\lambda_{j} c}{L_{x}}
$$

but

$\gamma_{m m}=\gamma_{p m f}\left\{1-\frac{\Gamma_{a} L_{x} L_{y}}{2}\left[H_{b}(j, k)-\frac{c^{2} H_{a}(j, k)}{\gamma_{p m f}^{2}}\right]\right\}=\frac{\lambda_{j} c}{L_{x}}$.

Equations (90) and (92) show that for the same natural frequency, the critical speed for the same system consisting of an isotropic rectangular plate resting on a constant foundation and traversed by a uniformly distributed moving force, is greater than that traversed by a uniformly distributed moving mass. Thus, resonance is reached earlier in the moving distributed mass system than in the moving distributed force system.

\section{Numerical Results and Discussion}

In order to illustrate the foregoing analysis, a rectangular plate of length $L_{x}=4.57 \mathrm{~m}$ and width $L_{y}=9.14 \mathrm{~m}$ is considered. The mass per unit length of the plate is $\mu=2758.291 \mathrm{Kg} / \mathrm{m}$, mass ratio $\Gamma_{1}=0.2$, and bending rigidity $D=10000$. The velocity of the travelling distributed load is $c=1.5 \mathrm{~m} / \mathrm{s}$. The values of the foundation modulus $K$ are varied between $0 \mathrm{~N} / \mathrm{m}^{3}$ and $4000000 \mathrm{~N} / \mathrm{m}^{3}$ and the values of the rotatory inertia correction factor $R_{0}$ are varied between 0.05 and 9.5.

In Figure 3, the transverse displacement response of the simple-clamped rectangular plate to moving distributed forces for various values of Foundation modulli $K$ and fixed value of rotatory inertia correction factor $R_{0}=.05$ is displayed. It is seen from the figure that as the values of the foundation modulli increase, the response amplitude of the simple-clamped rectangular plate under the action of distributed forces decreases. The same results are obtained when the same simple-clamped rectangular plate is traversed by moving distributed masses as depicted in Figure 5 . The response of the simple-clamped rectangular plate to moving distributed forces for various values of rotatory inertia correction factor $R_{0}$ and fixed value of foundation modulli $K=4000$ is shown in Figure 4. It is seen that the displacement of the plate decreases with increase in the rotatory inertia correction factor. The same behaviour characterizes the deflection profile of the same simple-clamped rectangular plate when it is traversed by moving distributed masses as shown in Figure 6. In Figure 7, the transverse displacement response of the clamped-clamped rectangular plate to

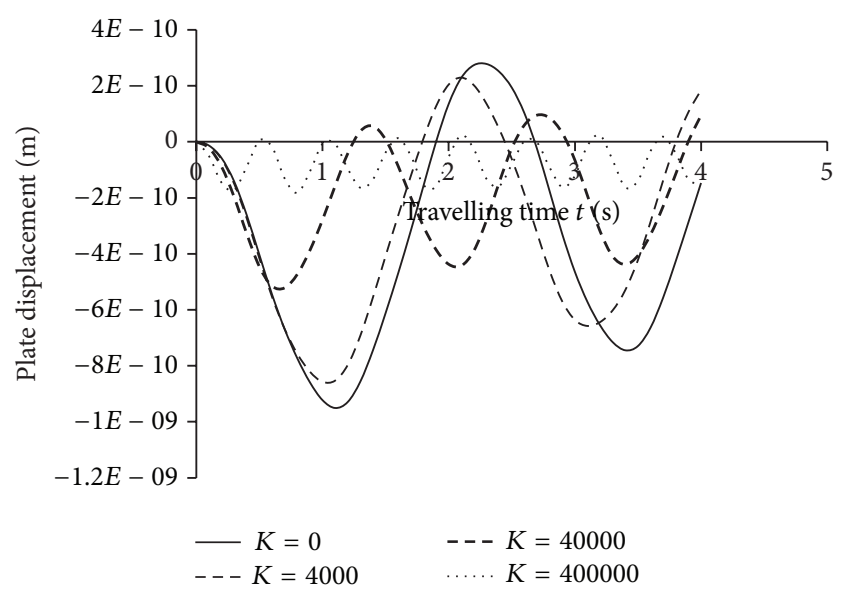

FIGURE 3: Displacement response to distributed forces of simpleclamped plate for various values of foundation moduli $K$.

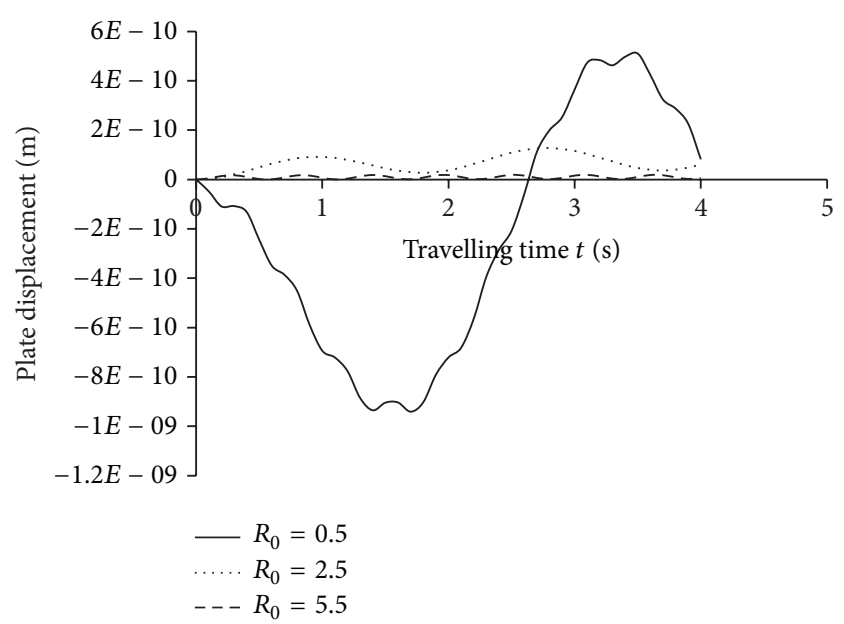

FIGURE 4: Displacement response to distributed forces of simpleclamped plate for various values of rotatory inertia correction factor $R_{0}$.

moving distributed forces for various values of Foundation modulli $K$ and fixed value of rotatory inertia correction factor $R_{0}=.05$ is displayed. It is seen from the figure that as the values of the foundation modulli increase, the response amplitude of the clamped-clamped rectangular plate under the action of distributed forces decreases. The same behaviour characterizes the displacement profile of the same clampedclamped rectangular plate when it is traversed by moving distributed masses as shown in Figure 9. The response of the clamped-clamped rectangular plate to moving distributed forces for various values of rotatory inertia correction factor $R_{0}$ and fixed value of foundation modulli $K=4000$ is shown in Figure 8. It is seen that the displacement of the plate decreases with increase in the rotatory inertia correction factor.

In Figure 10, the transverse displacement response of the clamped-clamped rectangular plate to moving distributed masses for various values of rotatory inertia correction factor 


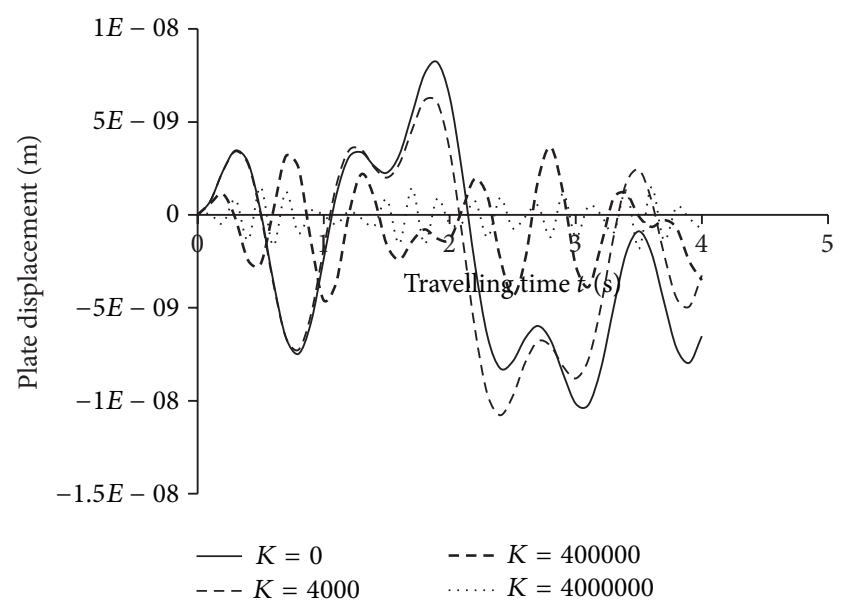

Figure 5: Displacement response to distributed masses of simpleclamped plate for various values of foundation moduli $K$.

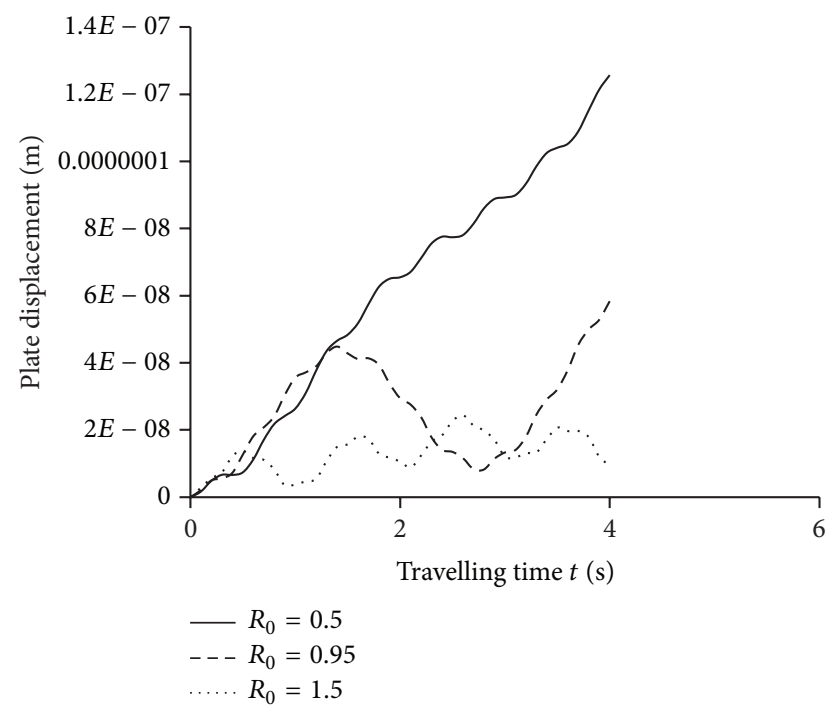

Figure 6: Displacement response to distributed masses of simpleclamped plate for various values of rotatory inertia correction factor $R_{0}$.

$R_{0}$ and fixed value of foundation modulli $K=4000$ is shown. It is seen that the displacement of the plate decreases with increase in the rotatory inertia correction factor.

Figure 11 depicts the comparison of the transverse displacement response for moving distributed force and moving distributed mass cases of the simple-clamped rectangular plate for fixed values of foundation modulli $K=4000$ and rotatory inertia correction factor $R_{0}=.05$. Clearly, the response amplitude of the moving distributed mass is greater than that of the moving distributed force problem. While Figure 12 depicts the comparison of the transverse displacement response for moving distributed force and moving distributed mass cases of the clamped-clamped rectangular plate for fixed values of foundation modulli $K=4000$ and rotatory inertia correction factor $R_{0}=.05$, it is observed

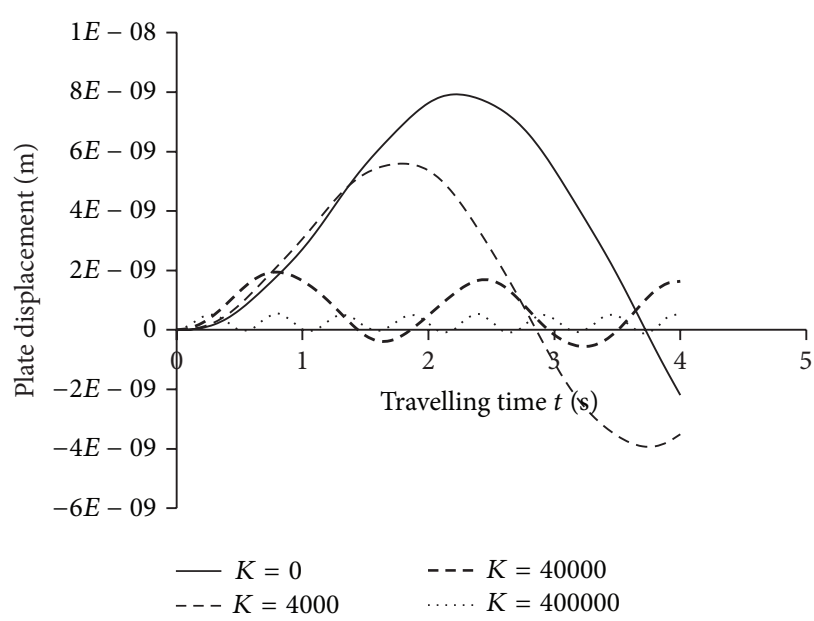

FIgURE 7: Displacement response to distributed forces of clampedclamped plate for various values of foundation moduli $K$.

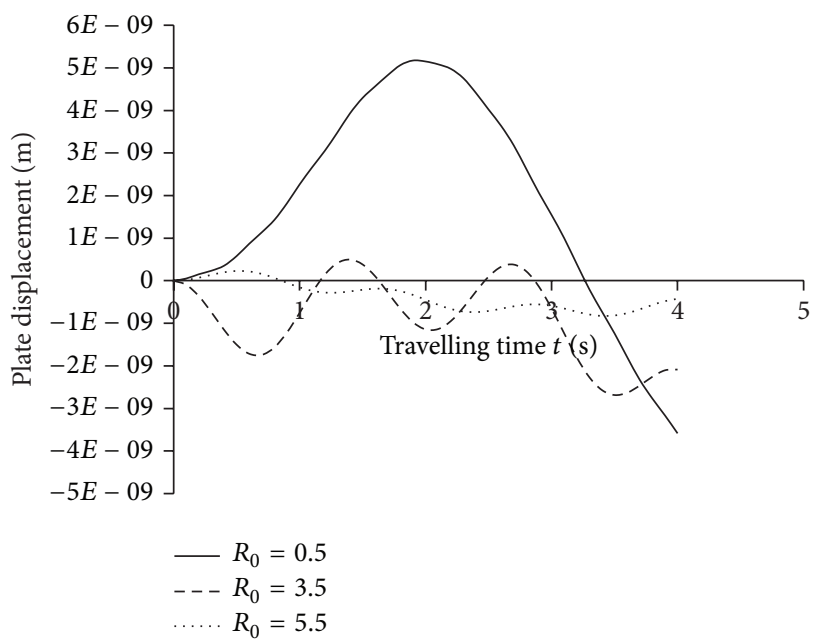

FIGURE 8: Displacement response to distributed forces of clampedclamped plate for various values of rotatory inertia correction factor $R_{0}$.

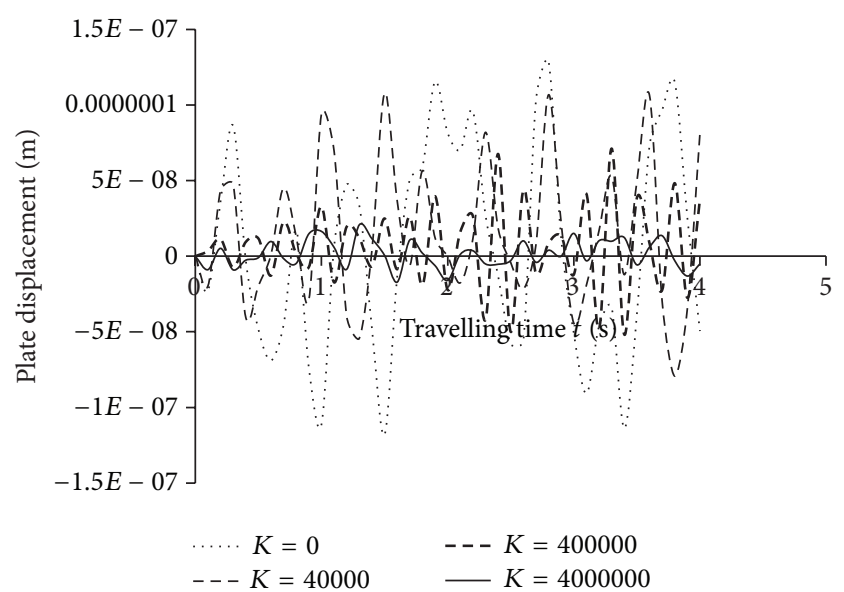

FIGURE 9: Displacement response to distributed masses of clampedclamped plate for various values of foundation moduli $K$. 


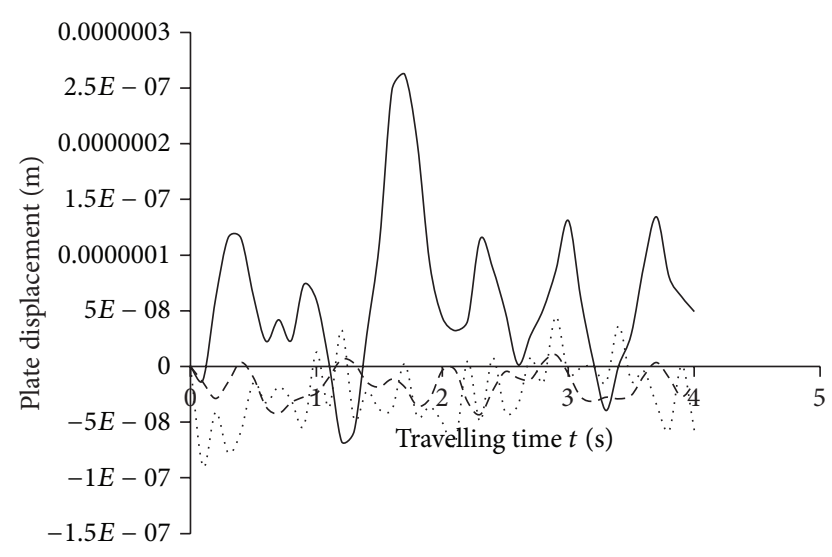

$$
\begin{aligned}
-R_{0} & =0.5 \\
\ldots . . R_{0} & =4.5 \\
---R_{0} & =9.5
\end{aligned}
$$

FIGURE 10: Displacement response to distributed masses of clampedclamped plate for various values of rotatory inertia correction factor $R_{0}$.

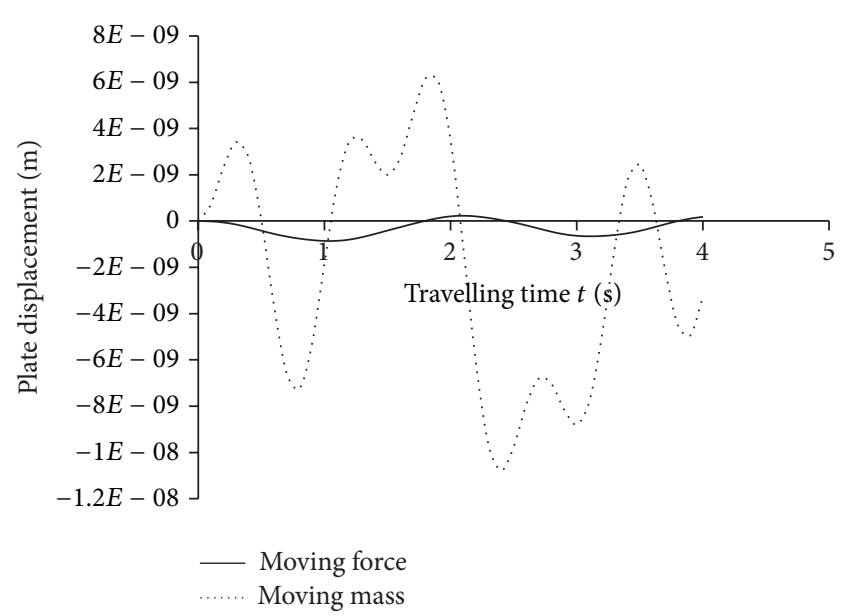

FIGURE 11: Comparison of displacement response of distributed force and distributed mass of simple-clamped rectangular plate for fixed values of $K=4000$ and $R_{0}=.05$.

that the response amplitude of the moving distributed mass is greater than that of the moving distributed force problem.

\section{Conclusion}

In this paper, the problem of the flexural vibrations of a rectangular plate having arbitrary supports at both ends is presented. The solution technique suitable for all variants of classical boundary conditions involves using the generalized two-dimensional integral transform to reduce the fourth order partial differential equation governing the vibration of the plate to a second order ordinary differential equation which is then treated with the modified asymptotic method of Struble. The closed form solutions obtained are analyzed and numerical analyses in plotted curves were presented. Results show that as the foundation moduli $K$ and rotatory

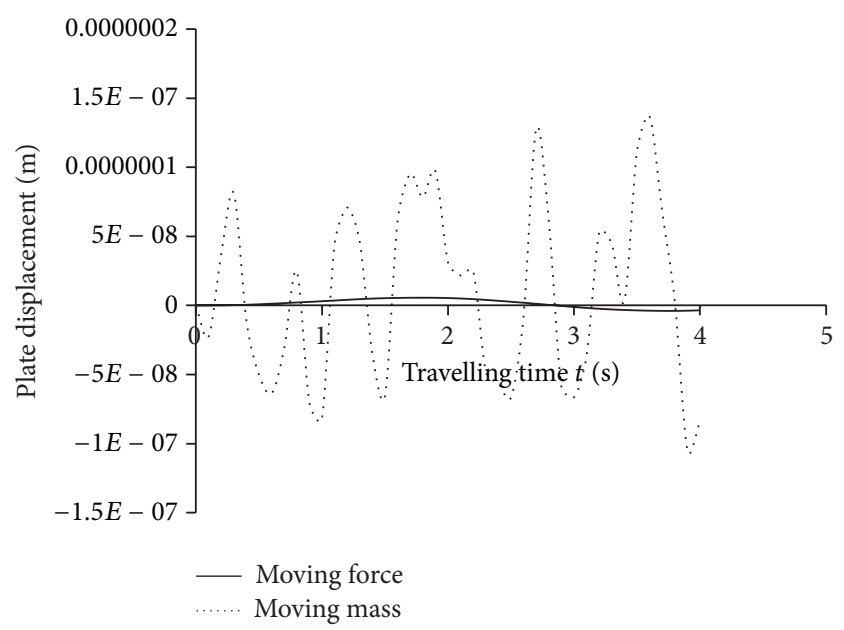

FIGURE 12: Comparison of displacement response of distributed force and distributed mass of clamped-clamped rectangular plate for fixed values of $K=4000$ and $R_{0}=.05$.

inertia correction factor $R_{0}$ increase, the response amplitudes of the dynamic system decrease for all illustrative examples considered. Analytical solutions further show that for the same natural frequency, the critical speed for the system traversed by uniformly distributed moving forces at constant speed is greater than that of the uniformly distributed moving mass problem for both clamped-clamped and simpleclamped end conditions. Hence, resonance is reached earlier in the moving distributed mass system. It is clearly seen that for both end conditions under consideration, the response amplitude of the uniformly distributed moving mass system is higher than that of the uniformly distributed moving force system for fixed values of rotatory inertia correction factor and foundation moduli. Thus, higher values of rotatory inertia correction factor and foundation moduli reduce the risk factor of resonance in a vibrating system.

\section{Conflict of Interests}

The authors declare that there is no conflict of interests regarding the publication of this paper.

\section{References}

[1] S. Sadiku and H. H. E. Leipholz, "On the dynamics of elastic systems with moving concentrated masses," Ingenieur-Archiv, vol. 57, no. 3, pp. 223-242, 1987.

[2] G. V. Rao, "Linear dynamics of an elastic beam under moving loads," Journal of Vibration and Acoustics, vol. 122, no. 3, pp. 281289, 2000.

[3] Y.-H. Chen and C.-Y. Li, "Dynamic response of elevated highspeed railway," Journal of Bridge Engineering, vol. 5, no. 2, pp. 124-130, 2000.

[4] E. Savin, "Dynamic amplification factor and response spectrum for the evaluation of vibrations of beams under successive moving loads," Journal of Sound and Vibration, vol. 248, no. 2, pp. 267-288, 2001. 
[5] S. T. Oni and T. O. Awodola, "Vibrations under a moving load of a non-uniform Rayleigh beam on variable elastic foundation," Journal of the Nigerian Association of Mathematical Physics, vol. 7, pp. 191-206, 2003.

[6] S. T. Oni and B. Omolofe, "Dynamic Analysis of a prestressed elastic beam with general boundary conditions under moving loads at varying velocities," Journal of Engineering and Engineering Technology, FUTA, vol. 4, no. 1, pp. 55-72, 2005.

[7] B. Omolofe and S. O. Ajibola, "On the transverse motions under heavy loads of thin beams with variable prestress," Journal of the Nigerian Association of Mathematical Physics, vol. 9, pp. 127-142, 2005.

[8] G. Muscolino and A. Palmeri, "Response of beams resting on viscoelastically damped foundation to moving oscillators," International Journal of Solids and Structures, vol. 44, no. 5, pp. 1317-1336, 2007.

[9] J. A. Gbadeyan and S. T. Oni, "Dynamic response to moving concentrated masses of elastic plates on a non-Winkler elastic foundation," Journal of Sound and Vibration, vol. 154, no. 2, pp. 343-358, 1992.

[10] S. T. Oni, "Flexural vibrations under Moving Loads of Isotropic rectangular plates on a Non-Winkler elastic foundation," Journal of the Nigerian Society of Engineers, vol. 35, no. 1, pp. 18-27, 2000.

[11] M.-H. Huang and D. P. Thambiratnam, "Deflection response of plate on winkler foundation to moving accelerated loads," Engineering Structures, vol. 23, no. 9, pp. 1134-1141, 2001.

[12] M. R. Shadnam, M. Mofid, and J. E. Akin, "On the dynamic response of rectangular plate, with moving mass," Thin-Walled Structures, vol. 39, no. 9, pp. 797-806, 2001.

[13] S. W. Alisjahbana, "Dynamic response of clamped orthotropic plates to dynamic moving loads," in Proceedings of the 13th World Conference on Earthquake Engineering, Paper no. 3176, Vancouver, BC, Canada, August 2004.

[14] J.-S. Wu, M.-L. Lee, and T.-S. Lai, "The Dynamic analysis of a flat plate under a moving load by the finite element method," International Journal for Numerical Methods in Engineering, vol. 24, no. 4, pp. 743-762, 1987.

[15] R.-T. Wang and T.-Y. Lin, "Random vibration of multi-span mindlin plate due to moving load," Journal of the Chinese Institute of Engineers, vol. 21, no. 3, pp. 347-356, 1998.

[16] J. A. Gbadeyan and M. S. Dada, "Dynamic response of plates on Pasternak foundation to distributed moving loads," Journal of Nigerian Association of Mathematical Physics, vol. 5, pp. 184200, 2001.

[17] M. S. Dada, Vibration analysis of elastic plates under uniform partially distributed moving loads [Ph.D. thesis], University of Ilorin, Ilorin, Nigeria, 2002.

[18] M. A. Usman, "Dynamic response of a Bernoulli beam on Winkler foundation under the action of partially distributed load," Nigerian Journal of Mathematics and Applications, vol. 16, no. 1, pp. 128-150, 2003.

[19] I. A. Adetunde, Dynamic analysis of Rayleigh beam carrying an added mass and traversed by uniform partially distributed moving loads [Ph.D. thesis], University of Ilorin, Ilorin, Nigeria, 2003.

[20] J. A. Gbadeyan and M. S. Dada, "Dynamic response of a Mindlin elastic rectangular plate under a distributed moving mass," International Journal of Mechanical Sciences, vol. 48, no. 3, pp. 323-340, 2006.
[21] N. D. Beskou and D. D. Theodorakopoulos, "Dynamic effects of moving loads on road pavements: a review," Soil Dynamics and Earthquake Engineering, vol. 31, no. 4, pp. 547-567, 2011.

[22] J. V. Amiri, A. Nikkhoo, M. R. Davoodi, and M. E. Hassanabadi, "Vibration analysis of a Mindlin elastic plate under a moving mass excitation by eigenfunction expansion method," ThinWalled Structures, vol. 62, pp. 53-64, 2013.

[23] L. Fryba, Vibrations of Solids and Structures Under Moving Loads, Noordhoff, Groningen, The Netherlands, 1972.

[24] A. H. Nayfey, Perturbation Methods, John Wiley \& Sons, New York, NY, USA, 1973. 

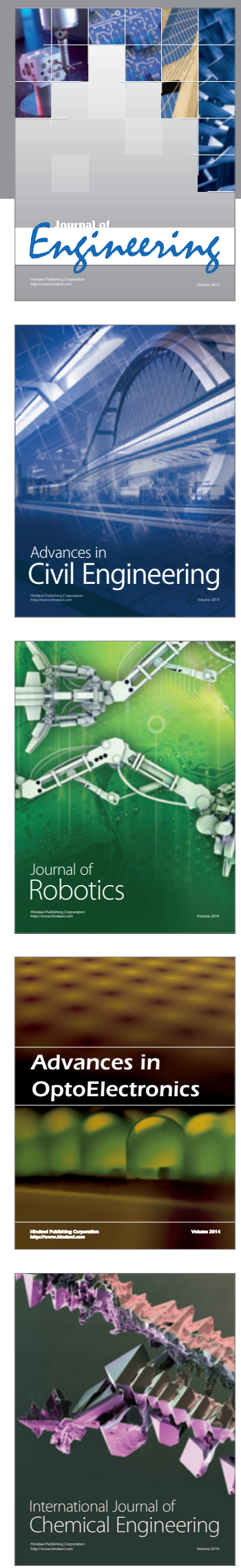

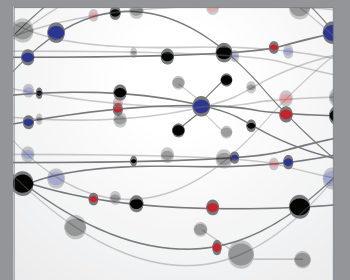

The Scientific World Journal
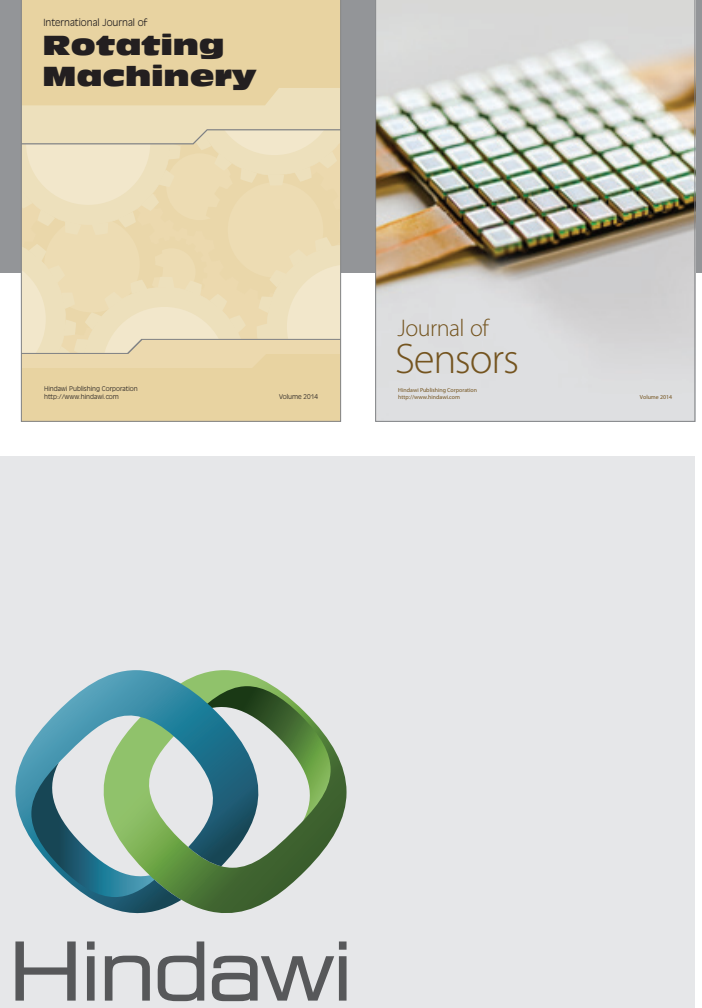

Submit your manuscripts at http://www.hindawi.com
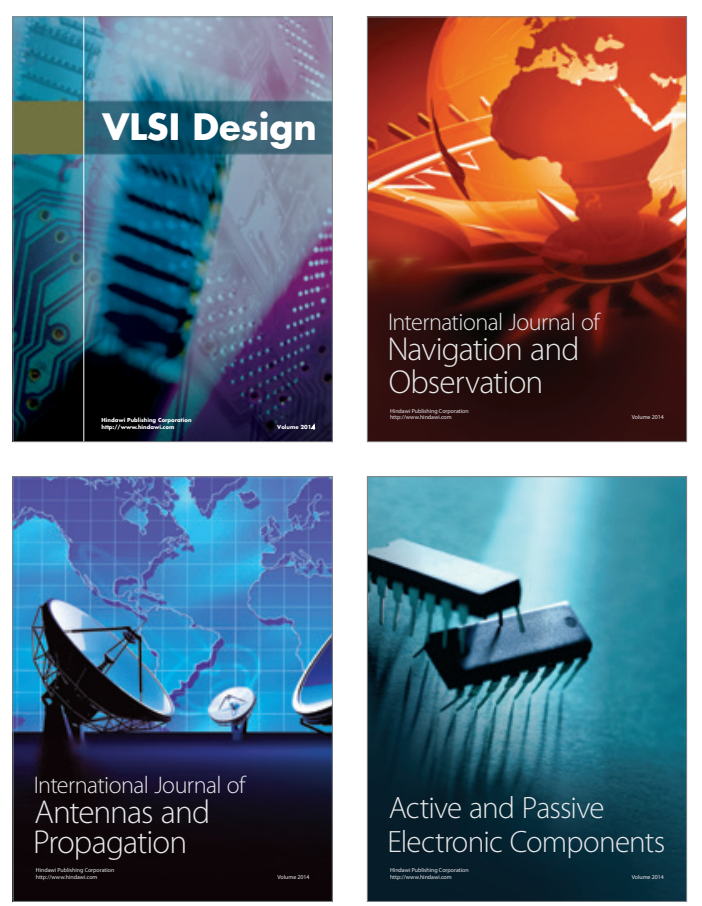
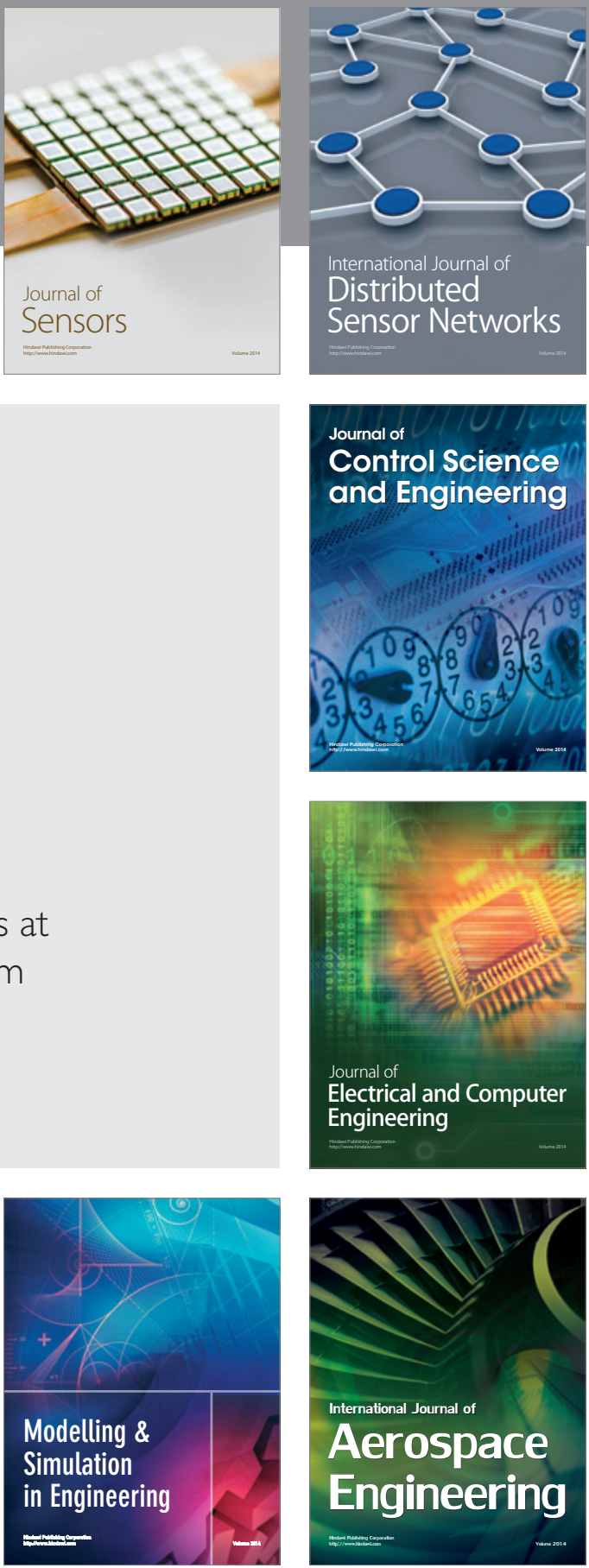

Journal of

Control Science

and Engineering
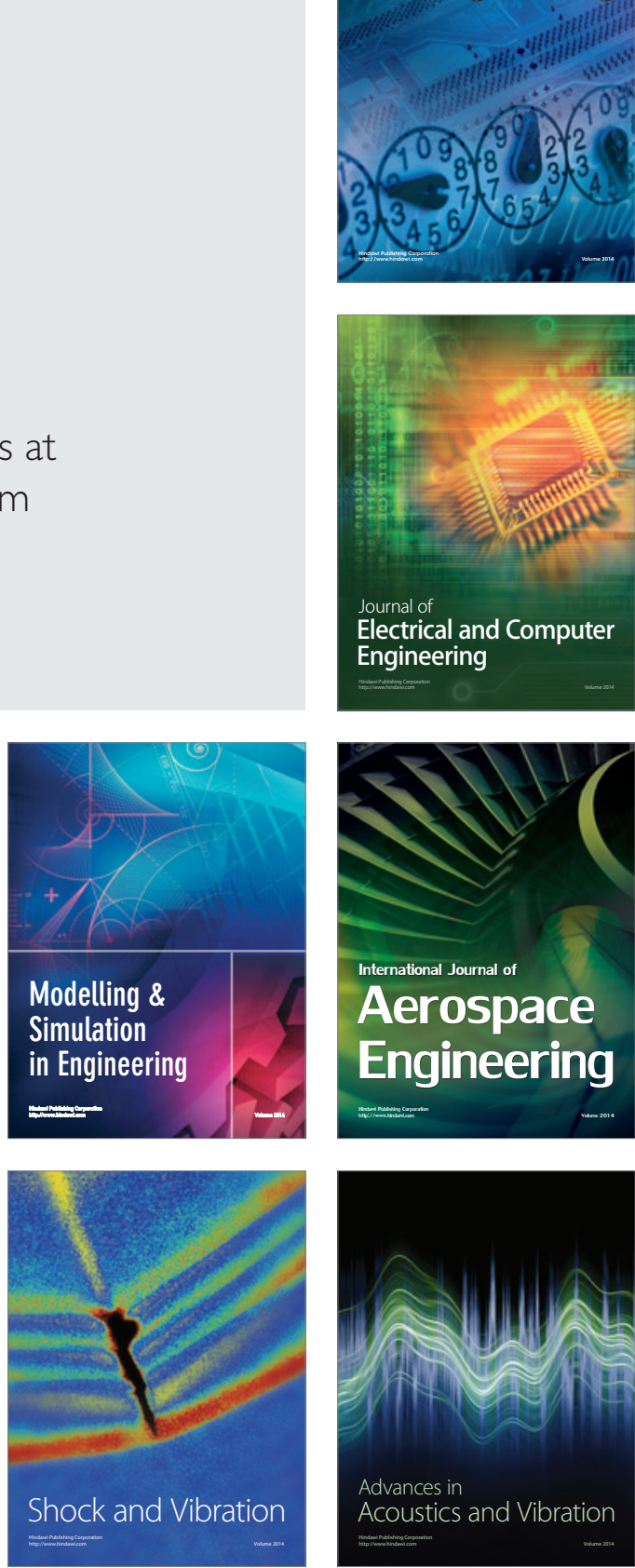